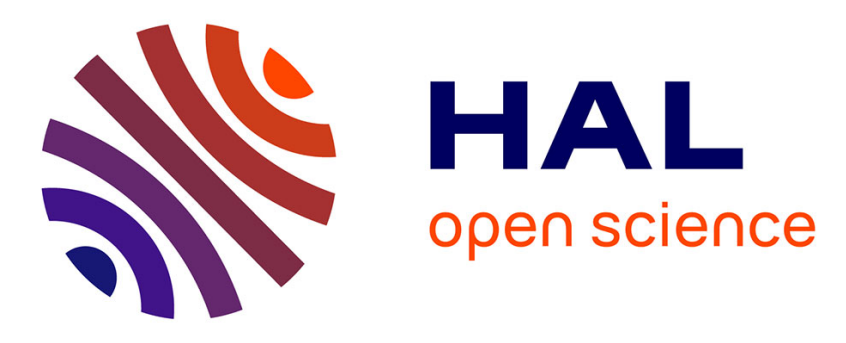

\title{
Towards Optimized MR Thermometry of the Human Heart at $3 \mathrm{~T}$
}

Silke Hey, Alexandru Cernicanu, Baudouin Denis de Senneville, Sébastien

Roujol, Mario Ries, Pierre Jais, Chrit Moonen, Bruno Quesson

\section{- To cite this version:}

Silke Hey, Alexandru Cernicanu, Baudouin Denis de Senneville, Sébastien Roujol, Mario Ries, et al.. Towards Optimized MR Thermometry of the Human Heart at 3T. NMR in Biomedicine, 2012, pp.35-43. hal-01063780

\section{HAL Id: hal-01063780 \\ https://hal.science/hal-01063780}

Submitted on 12 Sep 2014

HAL is a multi-disciplinary open access archive for the deposit and dissemination of scientific research documents, whether they are published or not. The documents may come from teaching and research institutions in France or abroad, or from public or private research centers.
L'archive ouverte pluridisciplinaire HAL, est destinée au dépôt et à la diffusion de documents scientifiques de niveau recherche, publiés ou non, émanant des établissements d'enseignement et de recherche français ou étrangers, des laboratoires publics ou privés. 


\section{Towards Optimized MR Thermometry of the Human Heart at 3T}

\begin{tabular}{|r|l|}
\hline Journal: & NMR in Biomedicine \\
\hline Wanuscript ID: & NBM-10-0093.R2 \\
\hline Diley - Manuscript type: & Research Article \\
\hline Complete List of Authors: & $\begin{array}{l}\text { Hey, Silke; Laboratory for Molecular and Functional Imaging - IMF } \\
\text { Cernicanu, Alexandru; Philips Healthcare } \\
\text { Denis de Senneville, Baudouin; Laboratory for Molecular and } \\
\text { Functional Imaging - IMF } \\
\text { Roujol, Sébastien; Laboratory for Molecular and Functional Imaging } \\
\text { - IMF } \\
\text { Ries, Mario; Laboratory for Molecular and Functional Imaging - IMF } \\
\text { Jaïs, Pierre; Hôpital Cardiologique du Haut-Lévêque } \\
\text { Moonen, Chrit; Laboratory for Molecular and Functional Imaging - } \\
\text { IMF } \\
\text { Quesson, Bruno; Laboratory for Molecular and Functional Imaging - } \\
\text { IMF }\end{array}$ \\
\hline Keywords: & \begin{tabular}{l} 
MR thermometry, cardiac arrhythmia, blood suppression \\
\hline
\end{tabular} \\
\hline
\end{tabular}




\section{Towards Optimized MR Thermometry of the Human Heart at 3T} Silke Hey*, Alexandru Cernicanu, Baudouin Denis de Senneville, Sébastien Roujol, Mario Ries, Chrit Moonen, Pierre Jaïs, Bruno Quesson



As a step towards PRFS-based thermometry of the human heart at 3T, the performance of two different MR thermometry sequences, as well as three different blood suppression techniques were evaluated on 8 healthy volunteers without heating. It was found that a temporal temperature standard deviation of $2^{\circ} \mathrm{C}$ or better can be achieved in the ventricular septum with an update rate equal to the cardiac frequency, when using a TFE-EPI sequence in combination with inflow saturation blood suppression, navigator respiratory compensation, and cardiac triggering combined with a motion compensation and multi-baseline correction algorithm for motion related susceptibility variations. 


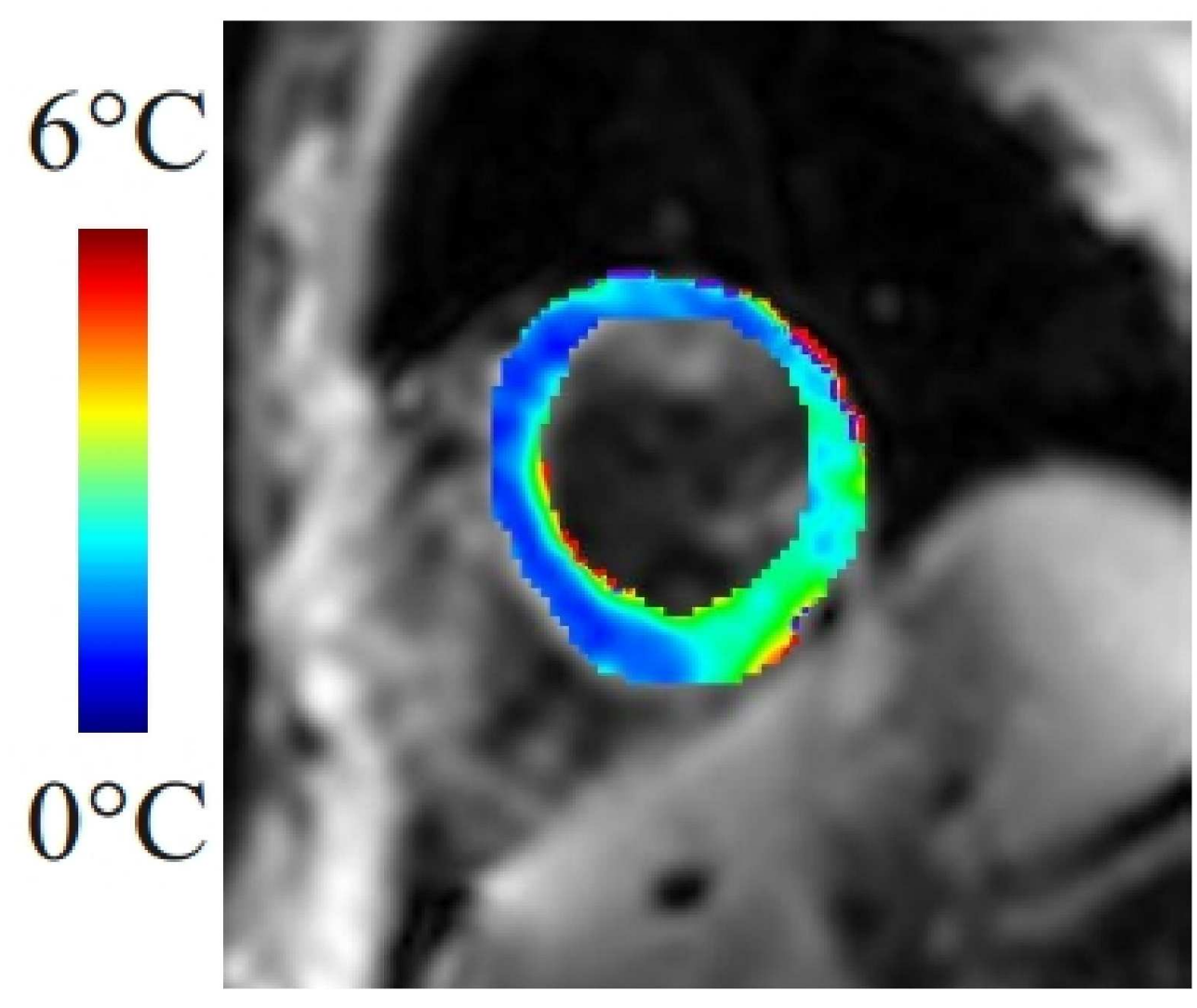

$135 \times 110 \mathrm{~mm}(600 \times 600 \mathrm{DPI})$ 


\title{
Towards Optimized MR Thermometry of the Human Heart at 3T
}

\author{
S. Hey ${ }^{1}$, A. Cernicanu ${ }^{2}$, B. Denis de Senneville ${ }^{1}$, S. Roujol ${ }^{1}$, M. Ries ${ }^{1}$, P. Jaïs ${ }^{3}$, \\ C.T.W. Moonen ${ }^{1}$, B. Quesson ${ }^{1}$ \\ ${ }^{1}$ Laboratory for Molecular and Functional Imaging, Bordeaux, France \\ ${ }^{2}$ Philips Healthcare, France \\ ${ }^{3}$ Hôpital Cardiologique du Haut-Lévêque, Bordeaux, France
}

\author{
address for correspondence: Silke Hey \\ Laboratory for molecular and functional imaging \\ UMR 5231/Université Bordeaux 2 \\ 146, rue Léo Saignat, Case 117 \\ 33076 Bordeaux Cedex, France \\ telephone : (+33) (0)5 57574592 \\ fax : (+33) (0)5 57574597 \\ silke.hey@imf.u-bordeaux2.fr
}

word count: 5217

short title: cardiac MR thermometry at $3 \mathrm{~T}$ 
Abstract

Catheter ablation using radio-frequency (RF) has been used increasingly for the treatment of cardiac arrhythmias. Proton resonance frequency shift (PRFS) based MR thermometry may provide intra-procedural feedback on the temperature distribution, helping with the determination of the therapy endpoint. As a step towards PRFS-based cardiac thermometry at 3T, we evaluated the suitability of two different MR thermometry sequences (TFE and TFE-EPI) and three blood suppression techniques using as a criterion the highest signal-to-noise (SNR) and contrast-to-noise ratios $(\mathrm{CNR})$, as well as the lowest temporal temperature standard deviation (corresponding to the highest temperature stability) and lowest temporal average of temperature in the myocardium. Experiments were performed without heating, using an optimized imaging protocol including navigator respiratory compensation, cardiac triggering, and image processing for the compensation of motion and susceptibility artifacts.

The effectiveness of blood suppression and its effect on temperature stability was evaluated in the ventricular septum of 8 healthy volunteers using multi-slice double inversion recovery (MDIR), motion-sensitized driven equilibrium (MSDE), and inflow saturation by saturation slabs (IS) over a period of 50 seconds of free breathing. It was shown that blood suppression during MR thermometry improves the CNR and the robustness of the applied motion correction algorithm. This was confirmed in the temperature stability. Furthermore, a gradient echo sequence with EPI readout acceleration and parallel imaging (SENSE) in combination with inflow saturation blood suppression was shown to achieve the best results. A temperature stability of $2^{\circ} \mathrm{C}$ or better in the ventricular septum, with a spatial resolution of $3.5 \times 3.5 \times 8 \mathrm{~mm}^{3}$, and a temporal resolution corresponding to the heart rate of the volunteer were observed.

Our results indicate that blood suppression is necessary and improves the temperature stability when performing cardiac MR thermometry. The proposed MR thermometry protocol, which optimizes temperature stability in the ventricular 
septum in the absence of heating, represents a step towards PRFS-based MR thermometry of the heart at $3 \mathrm{~T}$.

Key words: MR thermometry, cardiac arrhythmia, blood suppression

\author{
Abbreviations \\ PRFS - Proton Resonance Frequency Shift \\ TFE - Transient Field Echo \\ noBS - no Blood Suppression \\ MDIR - Multi-slice Double Inversion Recovery \\ MSDE - Motion Sensitized Driven Equilibrium \\ IS - Inflow Saturation \\ RF - Radio-frequency \\ SSFP - Steady State Free Precession \\ VCG - Vector-Cardiogram (uses 4 electrodes to map the ECG) \\ $\mathrm{T}_{\mathrm{I}} \quad$ - Inversion Time \\ $\mathrm{B}_{0} \quad$ - main magnetic field \\ $B_{1} \quad$ - Radio-frequency excitation field \\ $V_{\text {enc }} \quad$ - blood velocity corresponding to a phase accumulation of $\pi$ \\ $\sigma_{T} \quad$ - temperature stability (temporal temperature standard deviation at each \\ pixel) \\ $<\sigma_{\top}>\quad$ - spatial average of the temperature stability \\ $\mu_{\mathrm{T}} \quad$ - temporal mean of temperature \\ $\left\langle\mu_{\mathrm{T}}>\quad\right.$ - spatial average of the temporal mean of temperature \\ $\mathrm{p} \quad$ - significance level of the applied paired t-test
}




\section{Introduction}

Cardiac arrhythmias are amenable to catheter ablation using RF and even the most complex arrhythmias such as ventricular tachycardia and atrial fibrillation have been treated successfully [1, 2]. The established clinical methods for ablation catheter guidance are electroanatomic mapping (CARTO) and X-ray fluoroscopy [3]. In addition, echocardiography [4], computed tomography [5], and MR imaging [6] can be used separately or in combination $[7,8]$ to monitor the procedure. MRI can provide excellent soft-tissue contrast anatomical images, as well as real-time catheter visualization [9]. For pre- and post-procedural evaluation, MRI delayedenhancement sequences may be used to evaluate the substrate and the impact of the ablation [10]. Proton resonance frequency shift (PRFS) MR thermometry [11] with a dynamic series of phase images obtained with a gradient-echo based sequence was successfully used for the monitoring of radio-frequency ablation in other clinical contexts [12]. For cardiac ablation, MR thermometry and associated lethal thermal dose estimates could provide intra-procedural feedback. This may help with the determination of the cut-off point for the energy delivery and improve safety by avoiding damage to adjacent vital structures such as the esophagus or by preventing intra-tissular explosions and the associated risk of tamponades. In particular, information about the transmurality of the myocardial ablation may increase the success rate of the first ablation procedure.

However, due to the continued contraction and respiratory motion, cardiac imaging has to be rapid (acquisition time of less than $300 \mathrm{~ms}$ ) to avoid intra-scan motion and to allow for multi-slice imaging within one heartbeat. Typically used rapid steady state free precession sequences (SSFP) $[13,14]$ are prone to offresonance artifacts and require a high energy deposition, which can induce undesirable heating of the catheter wires [16]. Moreover, SSFP-based MR thermometry [15] is time-consuming and susceptible to motion artifacts limiting its applicability for monitoring cardiac RF ablations. To avoid these limitations, gradient echo sequences, potentially accelerated with EPI readouts and parallel imaging, represent interesting alternatives. 
Heart contraction and respiratory motion can be compensated with VCG-triggering and pencil-beam navigator-based slice tracking techniques, but additional image processing for the correct calculation of the temperature change at each pixel is required if residual in-plane motion is present $[17,18]$.

With an average thickness of the myocardial wall of 8 to $15 \mathrm{~mm}$ and the limited spatial resolution of MRI sequences, the contribution of blood signal resulting from partial volume effects at the interface between the cavity and the myocardium may affect MR thermometry. Blood suppression techniques could reduce this effect. The resulting increased contrast between the cavity and the myocardium may also be beneficial for the robustness of motion estimation algorithms, which are based on the analysis of local intensity changes.

In this paper, we evaluated the feasibility of $3 T$ PRFS MR thermometry of the myocardial wall using accelerated gradient-echo sequences adapted to cardiac imaging. We explored two options for PRFS MR cardiac thermometry sequences: gradient-echo with parallel imaging and EPI readout acceleration, and gradientecho with parallel imaging only. Furthermore, three different blood suppression techniques were tested: multi-slice double inversion recovery (MDIR) [19,20,21], motion-sensitized driven equilibrium (MSDE) [22-24], and inflow saturation (IS) [25]. An assessment of the applied sequences and the blood suppression techniques was performed on MR images from eight healthy volunteers using the contrast-to-noise (CNR) and signal-to-noise (SNR)-ratios. Finally, the temporal stability of PRFS MR thermometry [26] as well as the temporal average of temperature were evaluated.

\section{Materials and Methods}

\section{Choice of Imaging parameters}

The following imaging protocol has been optimized in order to meet certain requirements for cardiac thermometry in the myocardium:

- sufficient volume coverage with spatial resolution in the $\mathrm{mm}$ range to visualize 
the temperature distribution in the myocardium

- TE T2* for optimal temperature precision using PRF thermometry [27]

- a SNR of at least 10 to guarantee sufficient phase precision

- short acquisition time per slice (<300 ms) in order to avoid intra-scan motion

Parallel imaging acceleration (SENSE) was used in order to reduce the imaging matrix, but limited by the number of coil elements and signal considerations.

With acquisition times of $\sim 150 \mathrm{~ms}$ and $\sim 220 \mathrm{~ms}$ for TFE-EPI and TFE respectively, a minimum of 3 slices per cardiac cycle could be acquired without a compromise on the spatial resolution. Due to time constraints an in-plane resolution of $3.5 \mathrm{~mm}^{2}$ was chosen and signal considerations led to the selection of a $8 \mathrm{~mm}$ slice thickness. Even though fat suppression is necessary for PRF thermometry in organs like liver or kidney, this is not the case in the myocardium, as the fat content and chemical shift effects are negligible. However, the signal from subcutaneous fat in the field of view resulted in considerable artifacts in the center of the field of view due to the SENSE reconstruction. Therefore, fat suppression was applied for all experiments.

\section{Imaging protocol}

Imaging was performed on a $3 T$ MR system (Achieva, Philips, Best, The Netherlands) using a 6-channel surface cardiac coil. Respiratory through-plane motion was compensated with a slice-tracking technique that used a pencil-beam navigator placed on the right diaphragm to adjust the image slice position in realtime. All acquisitions were VCG-triggered, with a trigger delay $\left(T_{D}\right)$ set to the longest available value (between $109 \mathrm{~ms}$ and $699 \mathrm{~ms}$ ) depending on the used blood suppression technique and the cardiac frequency of the volunteer, ranging from 50 to 85 beats $/ \mathrm{min}$. For effective fat suppression in the presence of $B_{1}$ inhomogeneities, spectrally-selective adiabatic inversion recovery (SPAIR, inversion time $90 \mathrm{~ms}$ ) was applied before each slice acquisition. A RF-spoiled transient field echo (TFE) sequence $\left(T_{R}=7.3 \mathrm{~ms}, T_{E}=4.6 \mathrm{~ms}\right.$, flip angle $=26-30^{\circ}$, bandwidth/pixel $=291 \mathrm{~Hz}, \mathrm{FOV}=350 \times 280 \times 8 \mathrm{~mm}^{3}$, matrix $=100 \times 80$ ) with 
parallel imaging acceleration (SENSE) of 4 was tested.

In order to further accelerate the acquisition while maintaining a reasonable echo time for MR thermometry phase accumulation, a RF-spoiled TFE-EPI sequence $\left(T_{R}=11 \mathrm{~ms}, T_{E}=6 \mathrm{~ms}\right.$, flip angle $=26-30^{\circ}$, bandwidth $/$ pixel $=217 \mathrm{~Hz}, F O V=350$ $\times 350 \times 8 \mathrm{~mm}^{3}$, matrix $=100 \times 100$ ) with an echo train length of 3 and a SENSE factor of 3 was chosen in a second experiment. Echo train lengths of 5 or more were tested, but, despite the use of a localized main magnetic field $\left(B_{0}\right)$ shim on the heart, they led to significant image quality degradation on the heart and were not further used. In total, 100 consecutive short axis (mid position) images were acquired dynamically (dynamic scan time $t_{\text {dyn }}=0.75-1.2 \mathrm{~s}$ ) covering 3 slices per cardiac cycle (slice gap $0.8 \mathrm{~mm}$ ). The timing of the described sequence within the cardiac cycle is depicted in Fig. 1 a.

\section{Blood suppression techniques}

\section{Multi-slice double inversion recovery (DIR)}

The double inversion recovery (DIR) technique has been proven to reliably suppress the signal of blood, independent of the blood flow rate [19]. Nevertheless, the inversion delay severely prolongs the actual acquisition time and therefore DIR is generally limited to single-slice acquisitions, even though multislice implementations have been presented recently [20, 21].

In this work, a standard double inversion recovery (DIR) preparation module was adapted to multi-slice imaging (MDIR). As a compromise between volume coverage (a minimum of 3 slices) within the given cardiac cycle and blood suppression efficiency, inversion times $\left(T_{11} / T_{12}\right)$ of $4 \mathrm{~ms} / 320 \mathrm{~ms}$ were used.

\section{Inflow saturation (IS)}

For inflow saturation, saturation slabs positioned parallel to the image stack were used to suppress the signal of inflowing spins. IS is adapted to VCG-triggered 
multi-slice cardiac imaging, and was shown to provide efficient suppression of inflowing spins [25]. Here, inflow saturation (IS) was accomplished by placing one saturation slab of 60 to $80 \mathrm{~mm}$ thickness between the imaged slices and the base of the heart at a distance of 5 to $15 \mathrm{~mm}$ leading to a maximal increase in acquisition time of $6 \mathrm{~ms}$.

\section{Motion sensitized driven equilibrium (MSDE)}

The motion sensitized driven equilibrium magnetization preparation [22,23] has been evaluated and successfully used for black blood carotid imaging [24]. This approach only adds a minimal delay before the acquisition and is compatible with VCG-triggered cardiac multi-slice imaging. There is only a slight increase in scan time, but the preparatory module introduces $T_{2}$ weighting, which leads to additional signal loss. Moreover, MSDE may be sensitive to inhomogeneities of the RF excitation $\left(B_{1}\right)$ field, leading to a spatially varying flip angle, and eddy current effects, especially at high magnetic fields. The blood suppression MSDE preparatory module which was implemented for this work is detailed in Fig. 1b and represents an improved version of the module proposed in [28]. In order to achieve a higher robustness against $B_{1}$ inhomogeneities, two composite inversion pulses were used. Adiabatic pulses were tested. However, the resulting images showed motion artifacts as a result of the significantly increased duration of the preparation module and thus, non-adiabatic pulses were employed. Bipolar gradients were used in order to compensate for eddy current effects, with amplitudes calculated following the definition proposed by Nguyen et al [23]

$v_{\text {enc }}=\pi \gamma / m_{1}$ where $y$ is the gyromagnetic ratio, $m_{1}$ is defined as the first moment of the applied gradient, and $v_{\text {enc }}$ corresponds to the velocity of the blood spins which are dephased by $\pi$. This blood suppression technique is therefore velocity selective and an adaptation of $v_{\text {enc }}$ is required for each volunteer in order to match the blood velocity. The value of $v_{\text {enc }}$ was adjusted on each volunteer between 20 $\mathrm{cm} \cdot \mathrm{s}^{-1}$ and $40 \mathrm{~cm} \cdot \mathrm{s}^{-1}$ (corresponding to $\mathrm{m}_{1}$ of $16 \mathrm{mT} \cdot \mathrm{m}^{-1} \cdot \mathrm{ms}$ to $8 \mathrm{mT} \cdot \mathrm{m}^{-1} \cdot \mathrm{ms}$ ). It was adapted by varying the gradient strength while the length of the preparation 
module remained fixed at $9 \mathrm{~ms}$.

No precise adjustment of $v_{\text {enc }}$ for the three different encoding directions was performed as such an approach was considered too time-consuming.

The time penalties per image stack resulting from the application of one of the described blood suppression techniques were $320 \mathrm{~ms}$ for MDIR (corresponding to $\left.T_{12}\right), 9 \mathrm{~ms}$ for MSDE and $6 \mathrm{~ms}$ for IS.

\section{Image Processing}

\section{Motion compensation}

Despite the use of slice tracking and VCG triggering, which resulted in a substantial reduction of heart motion, residual in-plane displacements could be observed. In-plane motion compensation was performed by analyzing local displacements on the magnitude images. For this purpose, the first image in the time series was selected as the reference image, on which a region of interest (ROI) delimiting the left myocardium was manually drawn. A gradient driven descent algorithm maximizing the inter-correlation coefficient between this reference image and each following magnitude image of the time series was performed, assuming an affine displacement restricted to the selected ROI [17]. The 6 coefficients of the affine transformation (two translations, two scales, a rotation, a shear) leading to the highest correlation with the reference image were stored for each image of the time series. Images containing artifacts due to erroneous navigator slice tracking were identified by calculating the Pearson intercorrelation coefficient $\mathrm{C}_{0, \mathrm{n}}$ between each image and the first image of the time series. A rejection criterion was introduced such that images with $\mathrm{C}_{0, \mathrm{n}}<0.7$ were removed for further evaluation. In 7 of the 64 dynamic imaging series (100 images each), 2 to 27 images (on average 9 images) containing artifacts from erroneous slice tracking were detected and removed.

Evaluation of the image quality 
In order to evaluate the efficiency of the different blood suppression techniques SNR and CNR were evaluated from the motion compensated images. The inhomogeneous noise distribution in the images as a result of the applied parallel imaging technique required a modified definition for both expressions [24]

$$
\begin{aligned}
S N R & =\frac{S_{\mathrm{sep}}}{\sigma_{\mathrm{sep}}} \\
C N R & =\frac{S_{\mathrm{sep}}-S_{\mathrm{cav}}}{0.5\left(\sigma_{\mathrm{sep}}+\sigma_{\mathrm{cav}}\right)}
\end{aligned}
$$

where $S_{\text {sep }}$ denotes the average signal in a small $\mathrm{ROI}$ in the ventricular septum, $S_{\text {cav }}$ the average signal in a small $\mathrm{ROI}$ in the cavity and $\sigma_{\text {sep }}$ and $\sigma_{\text {cav }}$ the respective signal standard deviations in the same ROls. As a result, the calculated SNR allowed to draw conclusions about the ratio between the measured signal and the corresponding spatial variation of the signal in the chosen ROI, but not about the image noise in general.

\section{Temperature calculation}

Susceptibility effects were corrected with a model-based multi-baseline approach [17]. The first 50 images were acquired in a learning phase and the coefficients calculated during the registration were stored and used to create parametric phase maps assuming a linear relationship between respiratory motion and susceptibility related phase changes [29]. These parametric phase maps were computed by a Singular Value Decomposition (SVD) of the list of motion registered acquired phase images with the coefficients. For the following 50 images, the coefficients retrieved during the image registration and the parametric phase maps derived during the learning phase were used to compute a synthetic phase image. This synthesized reference phase was subtracted from the current phase to calculate the temperature map using the PRFS method [11]. In order to assess the quality of the temperature measurements in the myocardium, the temporal mean $\mu_{T}$ (reflecting systematic offsets) and standard deviation of temperature $\sigma_{T}$ (reflecting precision and stability) were calculated at each pixel. As rapid gradient echo- 
based sequences are prone to artifacts at the interfaces between myocardium, liver and lungs, the central region of the myocardium, corresponding to the ventricular septum, was chosen as target area for a detailed statistical analysis. The spatial distribution of $\sigma_{T}$, as well as the spatial average of $\sigma_{T}$ in a $R O I$ in the ventricular septum $\left\langle\sigma_{T}\right\rangle$, as well as the spatial distribution of the temporal mean of temperature $\mu_{T}$ and its spatial average $\left\langle\mu_{T}\right\rangle$, were analyzed. The aim of this evaluation was to find a combination of imaging sequence and blood suppression technique providing a minimal temporal standard deviation of temperature $\sigma_{T}$ (corresponding to a maximal temperature stability) as well as a minimal temporal mean of temperature $\mu_{\mathrm{T}}$. For this evaluation, all dynamic images of one slice for the 8 volunteers (in total 800 images) were used for each combination of acquisition sequence and blood suppression technique.

\section{Statistical Analysis}

The significance of the results for the SNR/CNR, the temperature stabilities, and temporal averages of temperature for the different blood suppression techniques was evaluated using ANOVA (analysis of variances) in form of a F-test-with significance threshold $p=0.05$. For the temperature stability and the temporal average of temperature, all voxels within the ventricular septum were taken into account and the average values across volunteers were compared between the different blood suppression techniques. The significance of the result for CNR and SNR was evaluated by comparing the average values across patients for each blood suppression method. If the test was found significant, additional paired ttests were applied to the data of all pairs of blood suppression techniques. A significance of $p=0.05$ was used and corrected with the Bonferroni method. This statistical analysis was performed slice by slice.

\section{Results}




\section{$\underline{\text { TFE sequence }}$}

\section{SNR/CNR and image quality}

Figure 2a-d show typical magnitude images of the central slice obtained on the same volunteer without blood suppression and using MDIR, MSDE and IS (from left to right). All three blood suppression techniques in combination with TFE showed a comparable good performance in terms of blood suppression efficiency and image quality. As a criterion for the evaluation of the blood suppression performance, the mean value over all volunteers of the temporal average of the CNR and SNR in the ventricular septum calculated according to equation (1) together with the corresponding temporal standard deviation are displayed in Fig. 3. The application of any of the three blood suppression techniques led to an increased CNR between the septum and the cavity without significant difference between the three blood suppression techniques ( $p>0.05)$. In the acquisitions without blood suppression, for 6 of 8 volunteers the myocardium appeared hypointense in comparison to the signal of the blood in the cavity leading to a negative CNR. For the SNR, no significant difference between the blood suppression techniques could be observed ( $p>0.05$ ). According to equation (1), a high SNR is an indication of reduced spatial signal variation in the septum as a result of the applied blood suppression. However, inflow saturation produced a much lower CNR in the first slice than in slices 2 and 3, while the other three blood suppression techniques delivered comparable CNR's for each slice. In all three slices, the application of blood suppression led to an increased CNR $(p<0.05)$.

\section{Temperature stability and temporal mean of temperature}

As a second comparison criterion, the results of the evaluation of the temperature stability and the temporal mean of temperature in the myocardium are shown in Figures $4 a-d$ and $5 a-d$ as an overlay on the magnified magnitude images for the central slice. Note that the distributions of the temperature stability and the temporal means of temperature were spatially inhomogeneous, showing a 
degradation at the intersection of the myocardium, liver, and lungs. However, at the interface between the lung and the myocardium and in the ventricular septum the temperature stability distribution and the distribution of the temporal mean of temperature remained stable. A detailed evaluation of the spatial distribution of the temperature stability in the ventricular septum is presented in Fig. 6 for the central slice using the combined data from all volunteers in form of Box-and-Whisker plots. In addition, the spatial average of the temperature stabilities in the ventricular septum including the corresponding spatial standard deviations are shown in Table 1 as the mean, minimal and maximal value over all volunteers for the different blood suppression techniques. Compared to the acquisition without blood suppression, the temperature stability was enhanced for IS and MSDE ( $p<$ $0.05)$, but did not show a significant improvement for MDIR $(p>0.05)$. Among the different blood suppression methods, MSDE achieved the best temperature stability $(p<0.05)$. In the cases with reduced temperature stability as compared to the results achieved without blood suppression, especially high deviations were found for MDIR for 2 volunteers, for MSDE for 3 volunteers, and for IS for 3 volunteers. No obvious correlation between the measured CNR in the ventricular septum as shown in Fig. 3 and the temperature stabilities were found.

Finally, Table 1 shows the spatial average and standard deviations of the temporal mean of temperature in the ventricular septum as the mean, minimal and maximal value over all volunteers for all blood suppression methods. On average, a positive temperature offset of $0.0^{\circ} \mathrm{C}$ to $0.7^{\circ} \mathrm{C}$ has been measured without a statistically significant difference between the blood suppression methods $(p>0.05)$.

For the two remaining slices the results for the temporal average of temperature were comparable. All blood suppression techniques showed a decreased temperature stability in the first and last slice.

In general, the average blood suppression performance of the three blood suppression techniques was comparable. 


\section{$\underline{\text { TFE-EPI sequence }}$}

\section{SNR/CNR and image quality}

The images acquired using MSDE and IS showed a comparable quality and blood suppression efficiency, whereas the image acquired with MDIR suffered from motion-induced blurring (Fig. 2e-h). MDIR and MSDE suffered from a reduced or comparable SNR in the ventricular septum as compared to the acquisition without blood suppression, while IS showed a higher SNR $(p<0.05)$ indicating that IS achieved a more favorable ratio between myocardial signal and spatial signal homogeneity than MDIR and MSDE (Fig. 3 right). As expected, the CNR between the myocardium and the cavity was increased for all blood suppression techniques, with the IS technique performing best in comparison with MDIR and MSDE $(p<0.05)$. Furthermore, in the acquisitions without blood suppression for 6 of 8 volunteers and for MSDE for 2 volunteers, the myocardium appeared hypointense in comparison to the signal of the blood in the cavity leading to a negative CNR, as illustrated in Fig. 3 displaying the average CNR. For the remaining slices (results not shown) the differences between the different methods were reduced and IS showed a decreased CNR in the first slice.

\section{Temperature stability and temporal mean of temperature}

Similar to the acquisitions using TFE without EPI readout, the temperature stability and temporal mean of temperature were deteriorated at the intersection of the myocardium, liver, and lungs, but improved in the other sections of the myocardium (Fig. 4e-h and 5e-h). Despite the apparent blurring in the magnitude images for MDIR, the measured temperature stability and temporal mean of temperature in the myocardium were not affected by these artifacts. A detailed statistical analysis of the spatial distribution of the temperature stability in the ventricular septum for the different blood suppression techniques is shown in Fig. 6 and Table 1 using the combined data from all volunteers and the central slice. Statistically significant improvements of the temperature stability in comparison to 
the acquisition without blood suppression could be observed for all three blood suppression techniques $(p<0.05)$. Unlike the acquisitions using TFE without EPI readout, IS blood suppression performed significantly better than MDIR and MSDE $(p<0.05)$, as confirmed in the Box-and-Whisker plots in Fig. 6, which represents the combined evaluation over the ROls of all volunteers. Among the cases with reduced temperature stability as compared to the acquisition without blood suppression, remarkable poor temperature stabilities were calculated for MDIR for 1 volunteer, and for MSDE for 3 volunteers. This manifested itself also in substantially decreased average temperature stabilities, as presented in Table 1, where for the mentioned cases values of up to $16.9^{\circ} \mathrm{C} \pm 22.9^{\circ} \mathrm{C}$ could be observed. For this sequence, there was a good correspondence between the observed CNR in the ventricular septum as displayed in Fig. 3 and the corresponding temperature stability for the majority of cases. This was confirmed in the results of MSDE for 2 volunteers, where a hypo-intense signal in the septum was coupled to a significantly reduced temperature stability. A similar observation could be made for 7 of 8 volunteers in the cases where no blood suppression was applied. On the other hand a significantly increased CNR for 4 volunteers was coupled to a very high temperature stability in the ventricular septum (data not shown).

Figure 7 shows an example graph of the temperature measured in three different characteristic points in the ventricular septum for the IS technique and volunteer 5, central slice. The temperature remained stable and was shifted by approximately $0.7^{\circ} \mathrm{C}, 1.5^{\circ} \mathrm{C}$ and $1.9^{\circ} \mathrm{C}$ for the three different points. The temperature graphs confirm that the temporal standard deviation of temperature increased for the points closer to the interface with the lungs and the liver as already stated above. In addition, Table 1 shows the spatial average and standard deviation of the temporal mean of temperature in the ventricular septum as the mean, minimal and maximal value over all volunteers for all blood suppression techniques. On average, mean temperatures of $0.2^{\circ} \mathrm{C}$ to $0.4^{\circ} \mathrm{C}$ have been measured for the different blood suppression techniques. The last acquired slice showed similar temperature stabilities and temporal averages of temperature as the central slice. 
For the first slice, the acquisitions with IS and MSDE produced lower temperature stabilities than for the second and third slice.

\section{Discussion}

\section{Imaging protocol}

A direct comparison of the two applied sequences (TFE and TFE-EPI) is not straightforward as several factors have to be taken into account. The TFE sequence was robust against motion artifacts and $B_{0}$ inhomogeneities while suffering from longer acquisition times. As a result, the achievable echo times were limited, leading to a reduced temperature precision. Combining this sequence with an EPI readout led to a significant acceleration and reduced intrascan motion, but the EPI readout was intrinsically more sensitive to susceptibility artifacts. The TFE-EPI sequence achieved on average better temperature stabilities in the ventricular septum, and enabled higher volume coverage and longer echo times.

The applied imaging protocol for MR thermometry required a compromise between spatial resolution, volume coverage, overall scan time and signal. In this study, a spatial resolution of $3.5 \times 3.5 \times 8 \mathrm{~mm}^{3}$ covering 3 slices was chosen in order to allow a temporal resolution of one heart beat, yielding an average SNR of 15 . Considering the size of a catheter tip and the expected hot spot during a RF ablation procedure, a higher spatial resolution would be desirable. As the SNR directly influences the theoretically achievable temperature precision [26], a further reduction of the voxel size would result in a decreased temperature precision, if the associated signal loss is not compensated by other means. A potentially higher signal could, for example, be achieved by using available 32-channel cardiac coils. Increasing the SENSE acceleration factor could be beneficial, but again, the resulting signal loss would have to be compensated in order to maintain a sufficient temperature precision. Furthermore, longer echo times, corresponding to potentially higher temperature precisions and increased volume coverage, could 
be achieved by selecting smaller fields of view (FOV). However, established inner volume excitation techniques [30,31] are usually based on inversion techniques which are not compatible with gradient echo imaging for MR thermometry. Other approaches using tailored RF pulses have been successfully tested, for example in 3D carotid imaging [32], but have not yet been applied to MR temperature imaging. The use of saturation slabs for the FOV reduction is possible with gradient echo sequences, but leads to an increased acquisition time.

\section{Blood suppression techniques}

IS: In terms of blood suppression, inflow saturation appeared to be the most robust method yielding a spatial average temperature stability in the ventricular septum of $2^{\circ} \mathrm{C}$ or better when combined with the TFE-EPI acquisition. Nevertheless, the application of inflow saturation slabs remained time-consuming and care had to be taken to avoid saturation of the spins included in the volume excited by the pencil beam. Furthermore, as IS is based on the inflow effect, the blood suppression efficacy in every slice depended on the distance of the saturation slab from the image stack, the exact blood velocity, and the slice order. In our setup, blood suppression was always more effective in the last acquired slice.

MDIR: Multi-slice DIR achieved good blood suppression over all slices leading to an average temperature stability of $3^{\circ} \mathrm{C}$ when used with a TFE sequence. However, it suffered from long scan times as a result of the necessary inversion delay limiting the slice number to 2 to 3 for typical cardiac frequencies (ranging from $0.8 \mathrm{~Hz}$ to $1.2 \mathrm{~Hz}$ for the presented volunteers). Furthermore, in our implementation, the combination with EPI readout led to severe image artifacts. This may be due to the fact that the EPI phase correction data was acquired prior to the inversion delay. It is acquired once at the beginning of a dynamic series of images by switching off the phase encoding gradients. However, if motion occurs between the acquisition of the phase correction data and the acquisition of the actual image, the resulting images will contain artifacts due to uncompensated residual phase offsets between the acquired k-space lines. A catheter tip in 
contact with the myocardium is likely to cause additional susceptibility artifacts, and hence lead to a further deterioration of the image quality [33]. If these problems are resolved, multi-slice DIR could become a candidate for blood suppression during MR thermometry using TFE sequences with or without EPI acceleration.

MSDE: The application of MSDE for blood suppression resulted in an average temperature stability of $3^{\circ} \mathrm{C}$ or better. However, due to its sensitivity to $\mathrm{B}_{0}$ and $\mathrm{B}_{1}$ inhomogeneities, large variations in the blood suppression performance were observed. This is also because for a chosen gradient strength, only one particular blood velocity is completely suppressed. As this technique also reduces the signal from all bulk motion, in some cases, the signal of the myocardium was reduced as well, leading to a degraded CNR between myocardium and cavity. General signal loss in the myocardium may be overcome by applying $B_{1}$ shimming [34] prior to the measurement, and by choosing different gradient amplitudes in the three encoding directions.

The results confirmed that blood suppression is beneficial for MR thermometry of the heart as indicated by the improved temperature stability in the myocardium. This is most likely due to a combination of reduced partial volume effects at the myocardium-blood interface and a better performance of the motion correction algorithms when presented with images with improved myocardium-cavity delineation. The observed average temperatures showed a large spatial variation and represent thus no systematic but random temperature offset.

Based on the average temperature stabilities and SNR/CNR values in the ventricular septum, IS in combination with TFE-EPI was the preferred combination for the presented implementation.

\section{Image Quality}

The measured SNR/CNR values in the ventricular septum indicated that the applied blood suppression was able to significantly increase the CNR between the 
myocardium and the cavity. It was also found that the application of blood suppression typically leads to an increased SNR in the ventricular septum. As all blood suppression methods are designed to cancel the signal of moving spins whilst also tissue spins may not remain completely unaffected, this result seems counterintuitive. This result may be attributed to a decreased spatial standard deviation of the signal, potentially as a result of reduced partial volume effects with blood and reduced flow artifacts.

\section{Motion correction}

The applied affine algorithm for the compensation of in-plane motion was still limited when applied in the myocardium, as already small deformations or residual flow effects could cause false pixel displacements in the myocardium. The temperature calculation during RF heating may be severely biased by the resulting false displacements. A local motion estimation algorithm [35] may be helpful, but may produce false corrections when presented with insufficient flow suppression. Hence, the development of more elaborated motion correction techniques will be the subject of future work. The removal of images containing artifacts represented a necessary processing step since without image rejection, temporal standard deviations of temperature above $20^{\circ} \mathrm{C}$ were measured.

The presented results for the temperature stability in the myocardium resulted from a combination of several effects including the quality of the motion and phase correction algorithms, the accuracy of the applied navigator slice tracking technique, and the efficiency of the applied blood suppression methods. As a result, they allowed only limited statements about the respective contributions of each parameter onto the temperature stability. Finally, the conclusions of this paper are based on the precision of MR thermometry in the absence of heating. Hence, future work should further explore the presented method during RF heating. 


\section{Conclusions}

PRFS-based cardiac MR-thermometry at $3 T$ using a TFE-EPI sequence with inflow saturation blood suppression could achieve average temperature stabilities of $2^{\circ} \mathrm{C}$ or better in the ventricular septum, in combination with cardiac triggering, navigator respiratory compensation, parallel imaging, and a motion compensation and multi-baseline susceptibility correction algorithm. For this setup, inflow saturation by saturation slabs appeared to be the blood suppression method of choice.

\section{Acknowledgments}

This work has been supported by the DiMI (Diagnostic Molecular Imaging) network and the author received a CIFRE stipend sponsored by ANRT (association nationale recherche technologie) and Philips Healthcare France. Finally, we thank Micheal McConnell (Stanford school of medicine) for helpful discussions.

\section{References}

[1] Chugh A, Morady F. Atrial fibrillation: Catheter ablation. J. Interv. Card. Electrophysiol. 2006; 16:15-26.

[2] Jaïs P, Cauchemez B, Macle L, Daoud E, Khairy P, Subbiah R, Hocini M, Extramiana $F$, Sacher $F$, Bordacher $P$, et al. Catheter ablation versus antiarrhythmic drugs for atrial fibrillation: The A4 study.

Circulation 2008; 118(24):2498-2505.

[3] Ector J, de Buck S, Huybrechts W, Nuygens D, Dymarkowski S, Bogaert J, Maes F, Heidbüchel $\mathrm{H}$. Biplane three-dimensional augmented fluoroscopy as single navigation tool for ablation of atrial fibrillation: Accuracy and clinical. Heart Rythm 2008; 5:957-964.

[4] Hijazi Z, Shivkumar K, Sahn D. Intracardial echocardiography during interventional and electrophysiological cardiac catheterization. 
Circulation 2009; 119:587-596.

[5] Lacomis M, Wigginton W, Fuhrman C, Schwarzman D, Armfeld R, Pealer K. Multi-detector row $\mathrm{CT}$ of the left atrium and pulmonary veins before radiofrequency catheter ablation for atrial fibrillation.

Radiographics 2003; 23:S35-S48.

[6] Dickfeld T. Magnetic resonance imaging and radiofrequency ablations. Herzschr. Elektrophys. 2007; 18:147-156.

[7] Malchano Z, Neuzil P, Cury R, Holmvang G, Weichet J, Schmidt E, Ruskin J, Reddy V. Integration of cardiac CT/MR imaging with three-dimensional electroanatomical mapping to guide catheter manipulation in the left atrium: Implications for catheter ablation of atrial fibrillation. J. Cardiovasc. Electrophysiol. 2006; 17:1221-1229.

[8] Saremi F, Tafti M. The role of computed tomography and magnetic resonance imaging in ablation procedures for treatment of atrial fibrillation.

Semin. Ultrasound CT MR 2009; 30(2):125-156.

[9] Schmidt E, Yoneyama R, Dumoulin C, Darrow R, Klein E, Kiruluta A, Hayase M. 3D coronary motion tracking in swine models with MR tracking catheters. J. Magn. Res. Imag. 2009; 29:86-98.

[10] Berruezo A, Ortiz-Peréz J, Guasch E, Tamborero D, Silva E, de Caralt T, Perea R, Boussy T, Mont L, Brugada J. Noninvasive evaluation of radiofrequency lesions in the human ventricular myocardium by contrastenhanced cardiac magnetic resonance. Circulation 2009; 2:208-11.

[11] Isihara Y, Calderon A, Watanabe H, Okamoto K, Suzuki Y, Kuroda K, Suzuki Y. A precise and fast temperature mapping using water proton chemical shift. Magn. Res. Med. 1992; 34(6):814-23.

[12] Seror, Lepetit-Coi $\square$ é M, Bail BL, de Senneville BD, Trillaud H, Moonen C, Quesson B. Real time monitoring of radiofrequency ablation based on MR thermometry and thermal dose in the pig liver in vivo.

Europ. Radiol. 2008; 18(2):408-16.

[13] Bundy J, Simonetti O, Laub G, Finn P. TrueFISP imaging of the heart. 
Proc. $7^{\text {th }}$ ISMRM, Berkeley (Calif), 1999. P 1282.

[14] Carr J, Simonetti O, Bundy J, Li D, Pereles S, Finn J. Cine MR angiography of the heart with segmented true fast imaging with steady-state precession. Radiology 2001; 219:828-834.

[15] Scheffler K. Fast frequency mapping with balanced SSFP: Theory and Application to Proton Resonance Frequency Shift Thermometry. Magn. Res. Med. 2004; 51:1205-1211.

[16] Nitz W, Oppelt A, Renz W, Manke C, Lenhart M, Link J. On the heating of linear conductive structures as guide wires and catheters in interventional MRI. J. Magn. Res. Imag. 2001; 13:105-114.

[17] Roujol S, Ries M, Quesson B, Moonen C, Denis de Senneville B. Real-time MR thermometry and dosimetry for interventional guidance on abdominal organs. Magn. Res. Med. 2010, 63(4):1080-1087

[18] Rieke V, Vigen K, Sommer G, Daniel B, Pauly J, Butts K. Referenceless PRF shift Thermometry. Magn. Res. Med. 2004; 51:1223-1231.

[19] Edelman R, Chien D, Kim D. Fast selective black blood MR imaging. Radiology 1991; 181:655-660.

[20] Song $H$, Wright A, Wolf $R$, Wehrli F. Multislice double inversion pulse sequence for efficient black blood MRI. Magn. Res. Med. 2002; 47:616-620.

[21] Yarnykh V, Yuan C. Multislice double inversion-recovery black-blood imaging with simultaneous slice reinversion. J. Magn. Res. Imag. 2003;17:478-483.

[22] Wang J, Yarnykh V, Hatsukami T, Chu B, Balu N, Yuan C. Improved suppression of plaque-mimicking artifacts in black-blood carotid atherosclerosis imaging using a multislice motion-sensitized drivenequilibrium (MSDE) turbo spin-echo (TSE) sequence. Magn. Res. Med. 2007; 58:973-981.

[23] Nguyen T, de Rochefort L, Spincemaille P, Cham M, Weinsaft J, Prince M, Wang Y. Effective motion-sensitizing magnetization preparation for black blood magnetic resonance imaging of the heart. J. Magn. Res. Imag. 2008; 28:1092-1100. 
[24] Koktzoglou I, Li D. Diffusion-prepared segmented steady-state free precession: Application to 3D black-blood cardiovascular magnetic resonance of the thoracic aorta and carotid artery walls.

J. Cardiovasc. Magn. Res. 2007; 9:33-42.

[25] Felmlee J, Ehman R. Spatial presaturation: A method for suppressing flow artifacts and improving depiction of vascular anatomy in MR imaging. Radiology 1987; 164:559-564.

[26] Weidensteiner C, Quesson B, Caire-Gana B, Kerioui N, Rullier A, Trillaud H, Moonen C. Real-time MR temperature mapping of rabbit liver in vivo during thermal ablation. Magn. Res. Med. 2003; 50(2):322-30.

[27] de Zwart JA, van Gelderen P, Kelly DJ, Moonen CTW. Fast magneticresonance temperature imaging. J. Magn. Res. B 1996;112(1):86-90

[28] Salerno M, Epstein F, Kramer C. Diffusion-prepared dark blood delayed enhancement imaging for improved detection of subendocardial infarcts. In Proc. 12th SCMR, 2009. Vol. II(Suppl I):O10.

[29] Roujol S, Denis de Senneville B, Maclair G, Hey S, Jaïs P, Moonen C, Quesson B. Advances in real-time MR temperature mapping of the human heart. Proc. $17^{\text {th }}$ ISMRM, Hawaii, 2009. P.443.

[30] Feinberg D, Hoenninger J, Crooks L, Kaufman L, Watts J, Arakawa M. Inner volume MR imaging: Technical concepts and their application. Radiology 1985; 156:743-747.

[31] Wilm B, Gramper U, Henning A, Pruessmann K, Kollias S, Boesiger P. Diffusion-weighted imaging of the entire spinal cord. NMR Biomed. 2009; 22(2):174-181.

[32] Bornstedt A, Bernhardt P, Hombach V, Kamenz J, Spiess J, Subgang A, Rasche V. Local excitation black blood imaging at 3T: Application to the carotid artery wall. Magn. Res. Med. 2008; 59:1207-1211.

[33] Kolandeivelu A, Zviman MM, Castro V, Lardo AC, Berger RD, Halperin HR. Non-invasive assessment of tissue heating during cardiac radiofrequency ablation using MRI thermography 
NMR in Biomedicine - For Peer Review Only

Page 27 of 65

$$
\begin{aligned}
& 1 \\
& 2 \\
& 3 \\
& 4 \\
& 5 \\
& 6 \\
& 7 \\
& 8 \\
& 9 \\
& 10 \\
& 11 \\
& 12 \\
& 13 \\
& 14 \\
& 15 \\
& 16 \\
& 17 \\
& 18 \\
& 19 \\
& 20 \\
& 21 \\
& 22 \\
& 23 \\
& 24 \\
& 25 \\
& 26 \\
& 27 \\
& 28 \\
& 29 \\
& 30 \\
& 31 \\
& 32 \\
& 33 \\
& 34 \\
& 35 \\
& 36 \\
& 37 \\
& 38 \\
& 39 \\
& 40 \\
& 41 \\
& 42 \\
& 43 \\
& 44 \\
& 45 \\
& 46 \\
& 47 \\
& 48 \\
& 49 \\
& 50 \\
& 51 \\
& 52 \\
& 53 \\
& 54 \\
& 55 \\
& 56 \\
& 57 \\
& 58 \\
& 59 \\
& 60
\end{aligned}
$$

Circ. Arrhythm. Electrophysiol. 2010. in press.

[34] Katscher U, Börnert P, Leussler C, van den Brink JS. Transmit sense. Man. Res. Med. 2003; 49:144-150.

[35] Denis de Senneville B, Mougenot C, Moonen C. Real time adaptive methods for treatment of mobile organs by MRI controlled high intensity focused ultrasound. Man. Res. Med. 2007; 57:319-330.

http://mc.manuscriptcentral.com/nbm 
Figure 1: a) Illustration of the TFE sequence using the MSDE blood suppression preparation including fat suppression and pencil-beam navigator. b) Positioning of the image acquisition within the cardiac cycle (RR-interval) for a pre-defined trigger delay (TD) and 3 slices leading to a dynamic scan time $t_{d y n}$.

Figure 2: Magnified magnitude images for TFE (a-d) and for TFE-EPI (e-h) for one volunteer, central slice. From left to right: without blood suppression (noBS), MDIR, MSDE, and IS.

Figure 3: Mean value over all volunteers of the temporal average of the SNR and CNR according to equation (1) together with the corresponding standard deviations for the different blood suppression techniques and the two different sequences for the central slice. Negative CNR values indicate a hypo-intense myocardial signal as compared to the signal of the blood in the cavity. The data was retrieved from ROl's in the ventricular septum using all 800 dynamic images for each combination of acquisition sequence and blood suppression technique. The size of the ROl's varied between 95 and 294 voxels with an average value of 175 voxels.

Figure 4: Magnified magnitude images with an overlay of the temperature stability $\sigma_{\mathrm{T}}$ for TFE (a-d) and for TFE-EPI (e-h) for one volunteer, central slice. From left to right: without blood suppression (noBS), MDIR, MSDE and IS. The data has been retrieved from the 100 dynamic images acquired for this specific volunteer.

Figure 5: Magnified magnitude images with an overlay of the temporal average of temperature $\mu_{\mathrm{T}}$ for TFE (a-d) and for TFE-EPI (e-h) for one volunteer, central slice. From left to right: without blood suppression (noBS), MDIR, MSDE and IS. The data has been retrieved from the 100 dynamic images acquired for this specific volunteer.

Figure 6: Box-and-Whisker plots of the spatial distribution of the temperature stability $\sigma_{T}$ in the ventricular septum for the acquisitions without blood suppression (no BS), DIR, MSDE and IS for the central slice. Shown are the values of the temperature stability which are exceeded by $10 \%, 25 \%, 50 \%, 75 \%$ and $90 \%$ of the voxels in the chosen ROI. The plots have been created from the combined data of all volunteers (in total 800 dynamic images). The size of the ROl's varied between 95 and 294 voxels with an average value of 175 voxels.

Figure 7: Example of the temperature evolution for TFE-EPI with IS for one volunteer, central slice. Shown are the graphs for three different voxels at characteristic locations in the ventricular septum as shown as white points on the magnitude image on the left. 


\begin{tabular}{|c|c|c|c|c|c|c|c|c|}
\hline & \multicolumn{4}{|c|}{ TFE } & \multicolumn{4}{|c|}{ TFE-EPI } \\
\hline & noBS & MDIR & MSDE & IS & noBS & MDIR & MSDE & IS \\
\hline$\left\langle\sigma_{\mathrm{T}}\right\rangle \quad\left[{ }^{\circ} \mathrm{C}\right]$ & $3.0 \pm 1.6$ & $3.1 \pm 1.8$ & $2.5 \pm 1.2$ & $2.8 \pm 1.7$ & $2.4 \pm 1.2$ & $2.0 \pm 0.9$ & $4.2 \pm 9.2$ & $1.6 \pm 0.6$ \\
\hline$<\sigma_{T}>_{\min }\left[{ }^{\circ} \mathrm{C}\right]$ & $1.7 \pm 0.3$ & $1.6 \pm 0.6$ & $1.4 \pm 0.4$ & $1.5 \pm 0.5$ & $1.6 \pm 0.4$ & $1.7 \pm 0.4$ & $1.4 \pm 0.3$ & $1.2 \pm 0.2$ \\
\hline$<\sigma_{T}>_{\max }\left[{ }^{\circ} \mathrm{C}\right]$ & $4.9 \pm 0.8$ & $6.2 \pm 1.8$ & $4.1 \pm 1.4$ & $4.8 \pm 2.4$ & $3.6 \pm 1.8$ & $3.6 \pm 0.9$ & $16.6 \pm 22.7$ & $2.3 \pm 0.9$ \\
\hline$\left\langle\boldsymbol{\mu}_{\mathrm{T}}\right\rangle \quad\left[{ }^{\circ} \mathrm{C}\right]$ & $0.4 \pm 1.1$ & $0.6 \pm 0.9$ & $0.0 \pm 1.0$ & $0.7 \pm 1.0$ & $0.2 \pm 0.9$ & $0.3 \pm 1.0$ & $0.5 \pm 13.5$ & $0.4 \pm 0.7$ \\
\hline$\left\langle\mu_{\mathrm{T}}\right\rangle_{\min }\left[{ }^{\circ} \mathrm{C}\right]$ & $-0.5 \pm 0.8$ & $-0.4 \pm 0.6$ & $-0.8 \pm 0.7$ & $-0.3 \pm 1.0$ & $-0.5 \pm 0.7$ & $-1.2 \pm 0.5$ & $-1.0 \pm 0.6$ & $-0.7 \pm 0.4$ \\
\hline$\left.<\mu_{\mathrm{T}}\right\rangle_{\max }\left[{ }^{\circ} \mathrm{C}\right]$ & $1.7 \pm 0.8$ & $1.5 \pm 0.5$ & $1.3 \pm 0.4$ & $1.7 \pm 0.7$ & $1.0 \pm 0.8$ & $1.5 \pm 0.6$ & $1.2 \pm 0.7$ & $1.1 \pm 0.3$ \\
\hline
\end{tabular}

Table 1: Spatial average of the temperature stability $\left\langle\sigma_{T}>\right.$ and the temporal average of temperature $\left\langle_{T}\right\rangle$ in the ventricular septum including the spatial standard deviations for all blood suppression techniques and the two tested sequences. Shown are the average values for all volunteers, as well as the minimal and maximal values found in the group of volunteers. This data is taken from the second slice. The size of the ROl's varied between 95 and 294 voxels with an average value of 175 voxels. 
Page 30 of 65

NMR in Biomedicine - For Peer Review Only

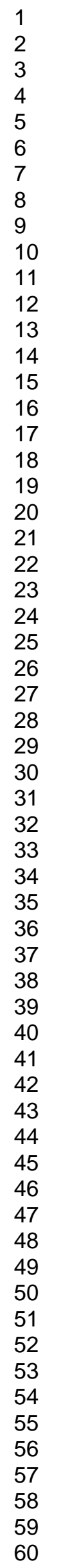

a)

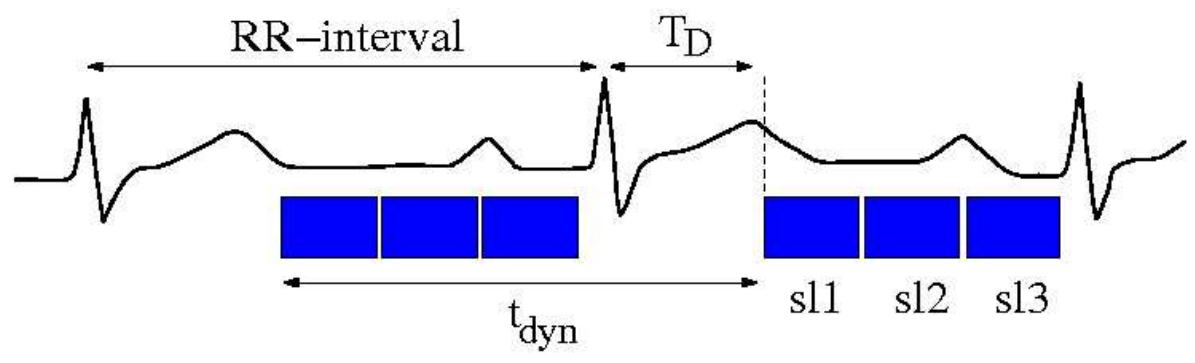

b)

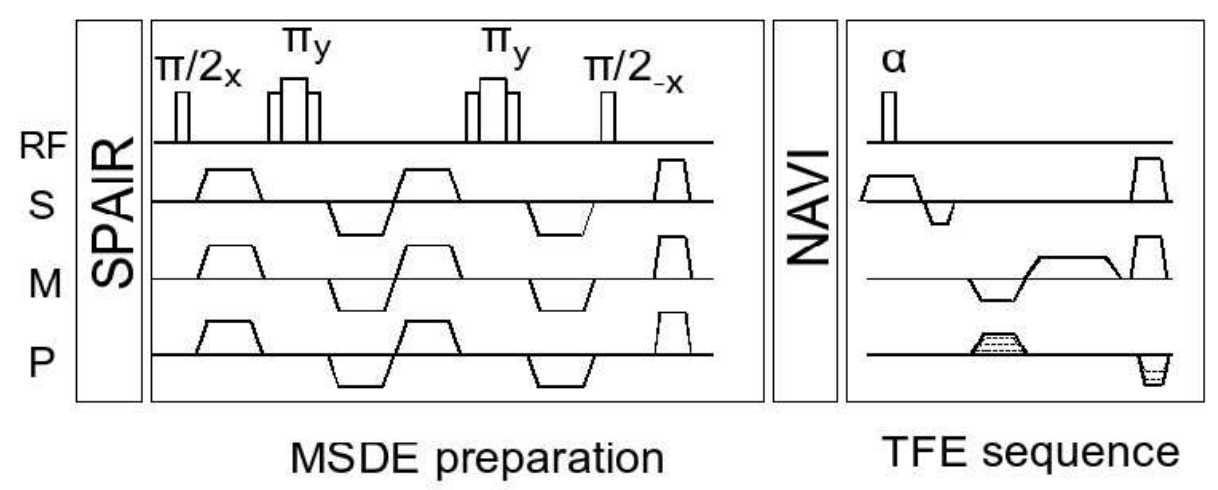

http://mc.manuscriptcentral.com/nbm 


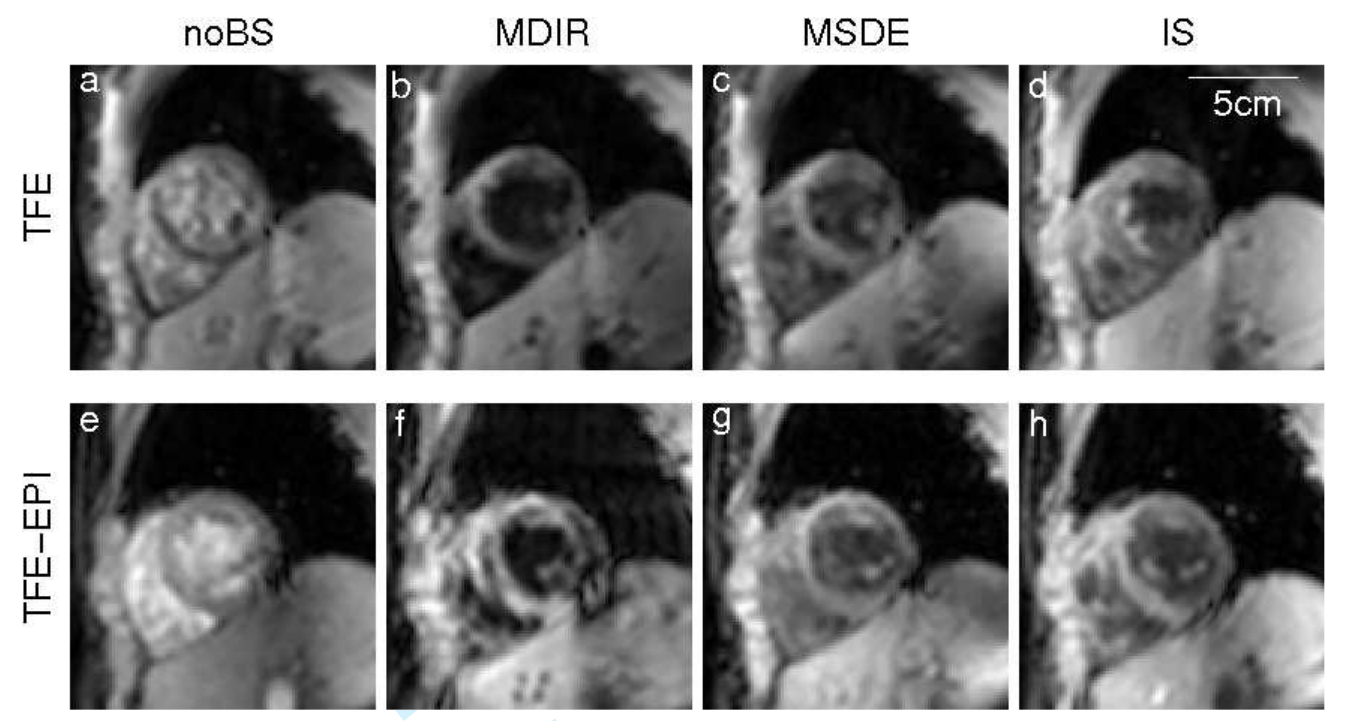


Page 32 of 65

NMR in Biomedicine - For Peer Review Only

1
2
3
4
5
6
7
8
9
10
11
12
13
14
15
16
17
18
19
20
21
22
23
24
25
26
27
28
29
30
31
32
33
34
35
36
37
38
39
40
41
42
43
44
45
46
47
48
49
50
51
52
53
54
55
56
57
58
59
60

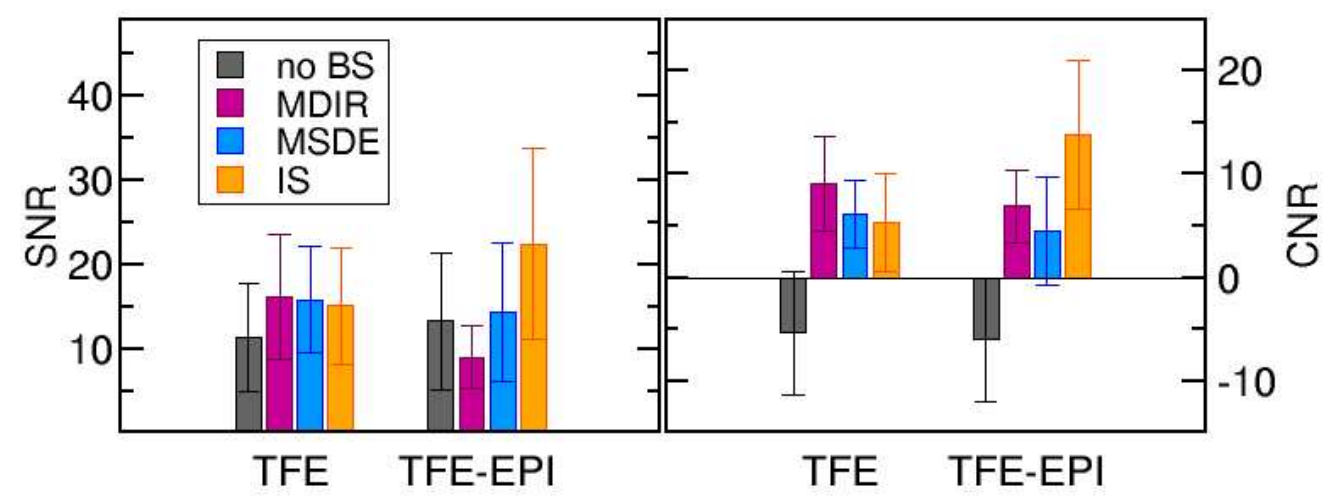

http://mc.manuscriptcentral.com/nbm 

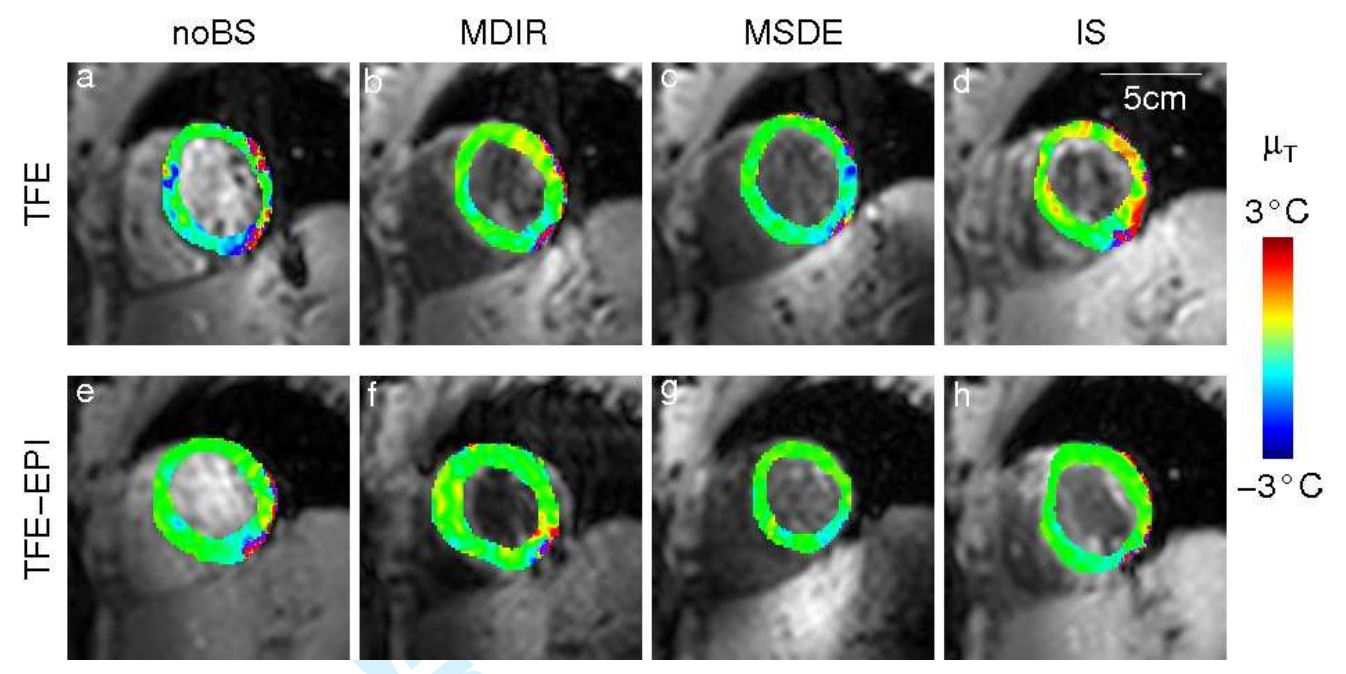


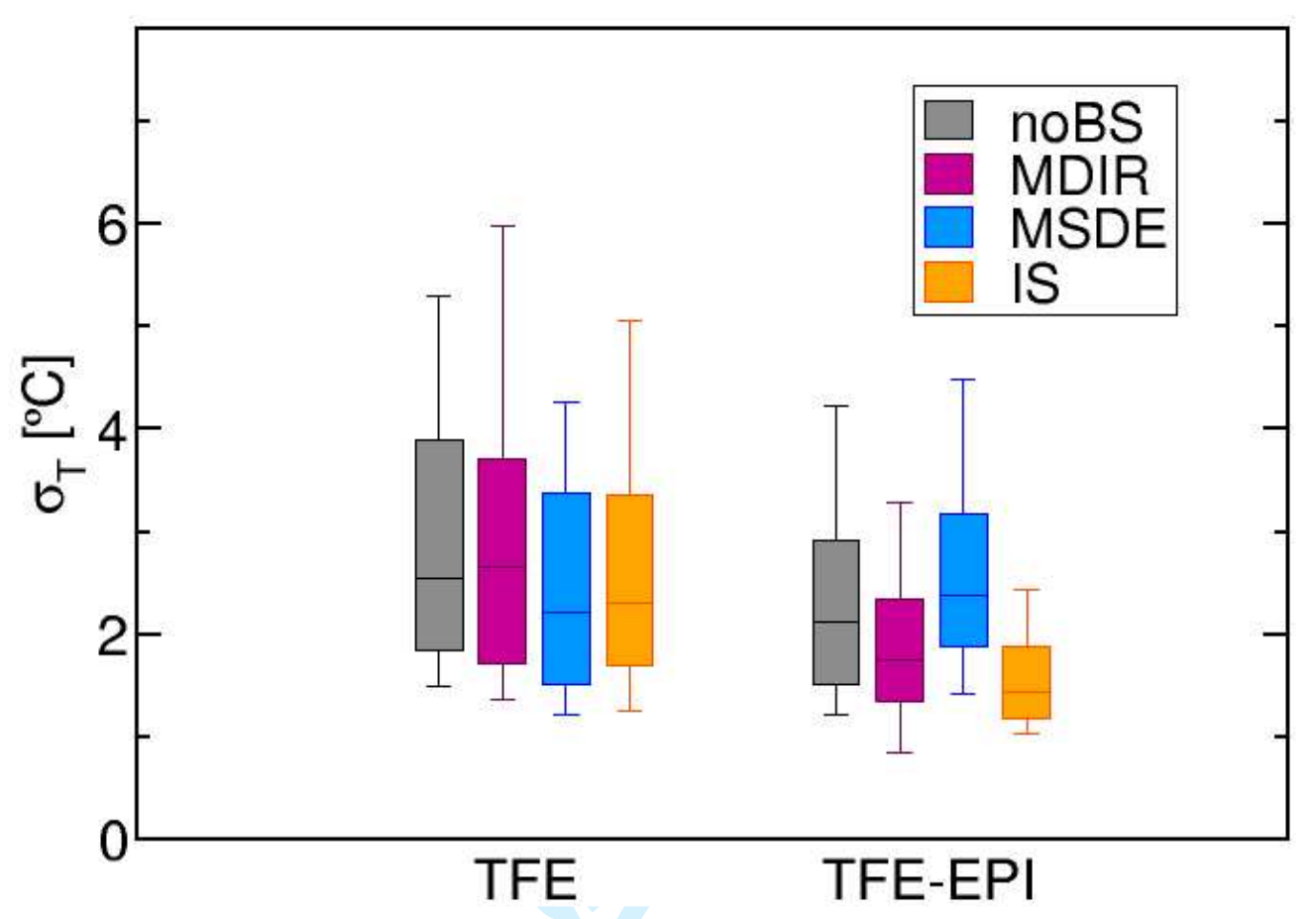

1

2

3

4

5

6
7

8

9

10

11

12

14

15

16

17

18

19

20

21

22

23

24

25

26

27

28

29

30

31

32

33

34

35

36

37

38

39

40

41

42

43

44

45

46

47

48

49

50

51

52

53

54

55

56

57

58

59

60 


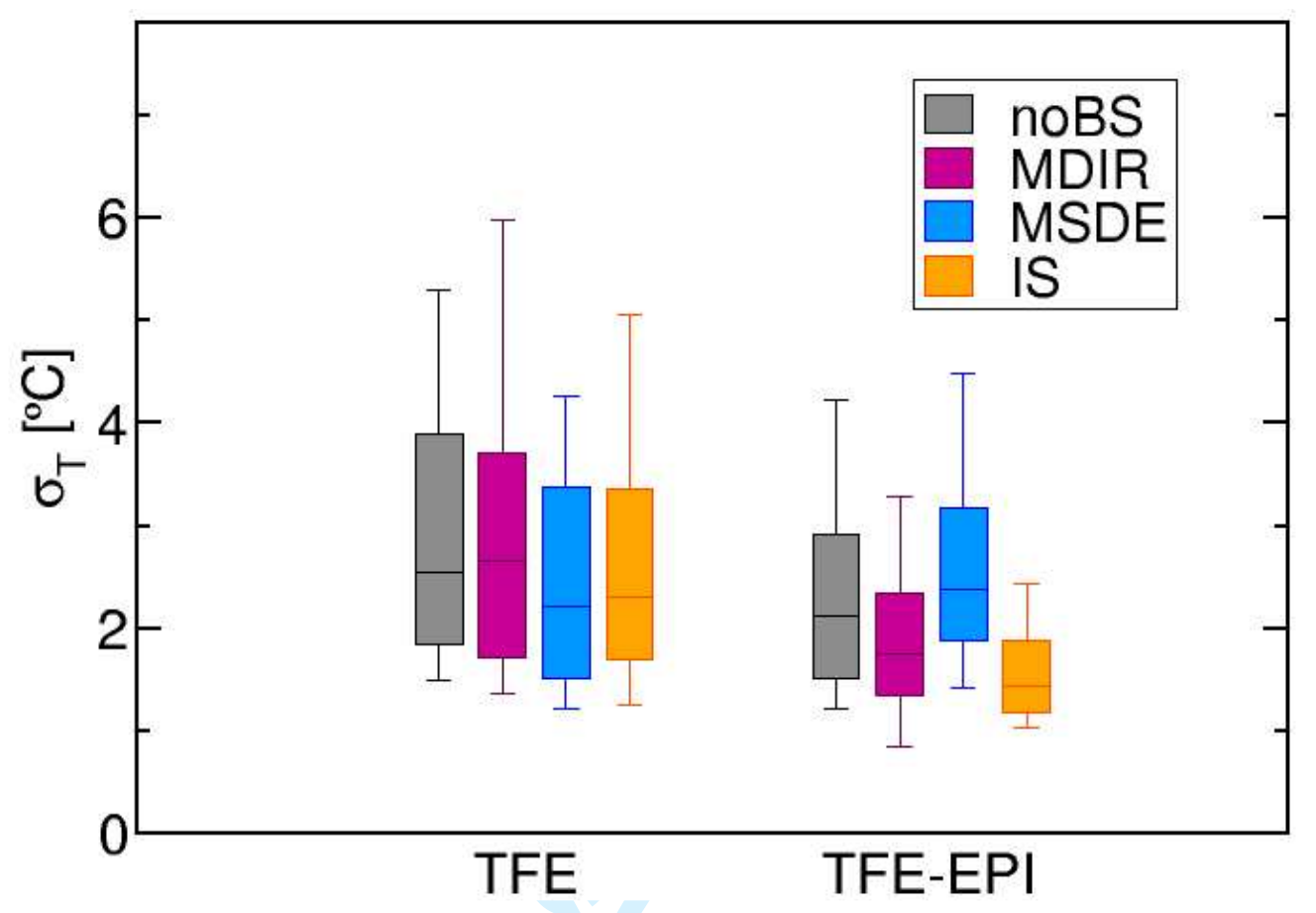

http://mc.manuscriptcentral.com/nbm 

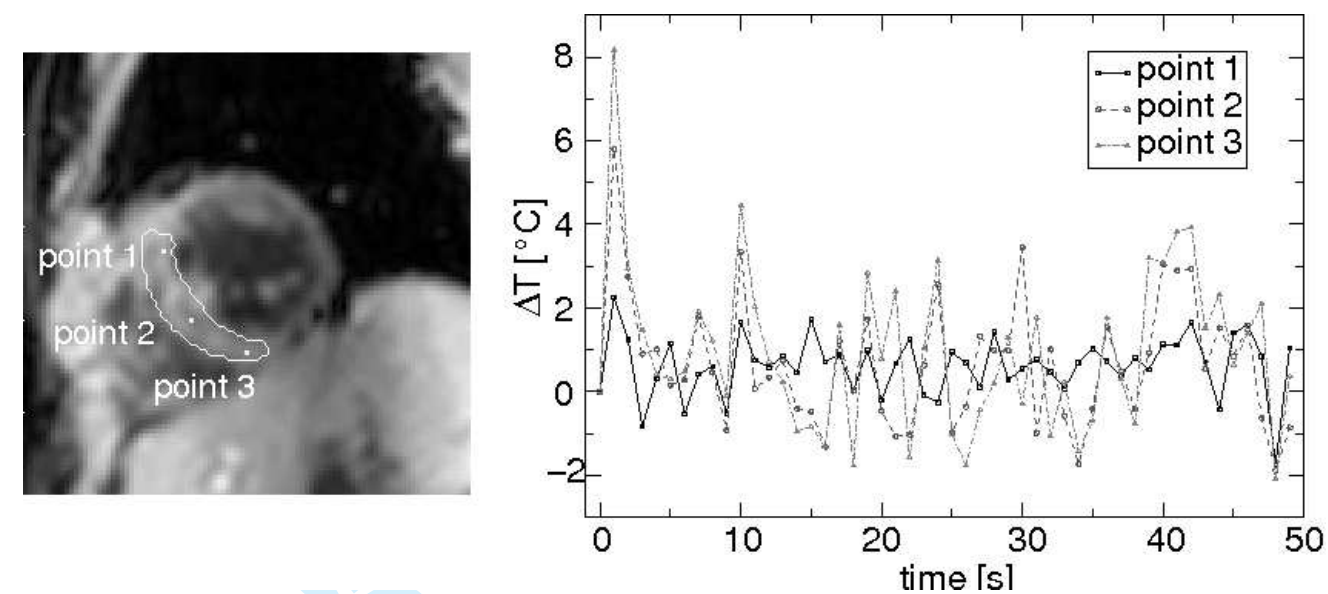

http://mc.manuscriptcentral.com/nbm 


\title{
Towards Optimized MR Thermometry of the Human Heart at 3T
}

\author{
S. Hey ${ }^{1}$, A. Cernicanu ${ }^{2}$, B. Denis de Senneville ${ }^{1}$, S. Roujol ${ }^{1}$, M. Ries ${ }^{1}$, P. Jaïs ${ }^{3}$, \\ C.T.W. Moonen ${ }^{1}$, B. Quesson ${ }^{1}$ \\ ${ }^{1}$ Laboratory for Molecular and Functional Imaging, Bordeaux, France \\ ${ }^{2}$ Philips Healthcare, France \\ ${ }^{3}$ Hôpital Cardiologique du Haut-Lévêque, Bordeaux, France
}

\author{
address for correspondence: Silke Hey \\ Laboratory for molecular and functional imaging \\ UMR 5231/Université Bordeaux 2 \\ 146, rue Léo Saignat, Case 117 \\ 33076 Bordeaux Cedex, France \\ telephone : (+33) (0)5 57574592 \\ fax : (+33) (0)5 57574597 \\ silke.hey@imf.u-bordeaux2.fr
}

word count: 5217

short title: cardiac MR thermometry at $3 T$ 
Abstract

Catheter ablation using radio-frequency (RF) has been used increasingly for the treatment of cardiac arrhythmias. Proton resonance frequency shift (PRFS) based MR thermometry may provide intra-procedural feedback on the temperature distribution, helping with the determination of the therapy endpoint. As a step towards PRFS-based cardiac thermometry at 3T, we evaluated the suitability of two different MR thermometry sequences (TFE and TFE-EPI) and three blood suppression techniques using as a criterion the highest signal-to-noise (SNR) and contrast-to-noise ratios (CNR), as well as the lowest temporal temperature standard deviation (corresponding to the highest temperature stability) and lowest temporal average of temperature in the myocardium. Experiments were performed without heating, using an optimized imaging protocol including navigator respiratory compensation, cardiac triggering, and image processing for the compensation of motion and susceptibility artifacts.

The effectiveness of blood suppression and its effect on temperature stability was evaluated in the ventricular septum of 8 healthy volunteers using multi-slice double inversion recovery (MDIR), motion-sensitized driven equilibrium (MSDE), and inflow saturation by saturation slabs (IS) over a period of 50 seconds of free breathing. It was shown that blood suppression during MR thermometry improves the CNR and the robustness of the applied motion correction algorithm. This was confirmed in the temperature stability. Furthermore, a gradient echo sequence with EPI readout acceleration and parallel imaging (SENSE) in combination with inflow saturation blood suppression was shown to achieve the best results. A temperature stability of $2^{\circ} \mathrm{C}$ or better in the ventricular septum, with a spatial resolution of $3.5 \times 3.5 \times 8 \mathrm{~mm}^{3}$, and a temporal resolution corresponding to the heart rate of the volunteer were observed.

Our results indicate that blood suppression is necessary and improves the temperature stability when performing cardiac MR thermometry. The proposed MR thermometry protocol, which optimizes temperature stability in the ventricular 
septum in the absence of heating, represents a step towards PRFS-based MR thermometry of the heart at 3T.

Key words: MR thermometry, cardiac arrhythmia, blood suppression

\author{
Abbreviations \\ PRFS - Proton Resonance Frequency Shift \\ TFE - Transient Field Echo \\ noBS - no Blood Suppression \\ MDIR - Multi-slice Double Inversion Recovery \\ MSDE - Motion Sensitized Driven Equilibrium \\ IS - Inflow Saturation \\ RF - Radio-frequency \\ SSFP - Steady State Free Precession \\ VCG - Vector-Cardiogram (uses 4 electrodes to map the ECG) \\ $\mathrm{T}_{\mathrm{I}} \quad$ - Inversion Time \\ $\mathrm{B}_{0} \quad-$ main magnetic field \\ $\mathrm{B}_{1} \quad$ - Radio-frequency excitation field \\ $v_{\mathrm{enc}} \quad$ - blood velocity corresponding to a phase accumulation of $\pi$ \\ $\sigma_{\mathrm{T}} \quad$ - temperature stability (temporal temperature standard deviation at each \\ pixel) \\ $\left.<\sigma_{\mathrm{T}}\right\rangle \quad$ - spatial average of the temperature stability \\ $\mu_{\mathrm{T}} \quad$ - temporal mean of temperature \\ $\left\langle\mu_{\top}\right\rangle \quad$ - spatial average of the temporal mean of temperature \\ p - significance level of the applied paired t-test
}




\section{Introduction}

Cardiac arrhythmias are amenable to catheter ablation using RF and even the most complex arrhythmias such as ventricular tachycardia and atrial fibrillation have been treated successfully [1, 2]. The established clinical methods for ablation catheter guidance are electroanatomic mapping (CARTO) and X-ray fluoroscopy [3]. In addition, echocardiography [4], computed tomography [5], and MR imaging [6] can be used separately or in combination [7, 8] to monitor the procedure. MRI can provide excellent soft-tissue contrast anatomical images, as well as real-time catheter visualization [9]. For pre- and post-procedural evaluation, MRI delayedenhancement sequences may be used to evaluate the substrate and the impact of the ablation [10]. Proton resonance frequency shift (PRFS) MR thermometry [11] with a dynamic series of phase images obtained with a gradient-echo based sequence was successfully used for the monitoring of radio-frequency ablation in other clinical contexts [12]. For cardiac ablation, MR thermometry and associated lethal thermal dose estimates could provide intra-procedural feedback. This may help with the determination of the cut-off point for the energy delivery and improve safety by avoiding damage to adjacent vital structures such as the esophagus or by preventing intra-tissular explosions and the associated risk of tamponades. In particular, information about the transmurality of the myocardial ablation may increase the success rate of the first ablation procedure.

However, due to the continued contraction and respiratory motion, cardiac imaging has to be rapid (acquisition time of less than $300 \mathrm{~ms}$ ) to avoid intra-scan motion and to allow for multi-slice imaging within one heartbeat. Typically used rapid steady state free precession sequences (SSFP) [13, 14] are prone to offresonance artifacts and require a high energy deposition, which can induce undesirable heating of the catheter wires [16]. Moreover, SSFP-based MR thermometry [15] is time-consuming and susceptible to motion artifacts limiting its applicability for monitoring cardiac RF ablations. To avoid these limitations, gradient echo sequences, potentially accelerated with EPI readouts and parallel imaging, represent interesting alternatives. 
Heart contraction and respiratory motion can be compensated with VCG-triggering and pencil-beam navigator-based slice tracking techniques, but additional image processing for the correct calculation of the temperature change at each pixel is required if residual in-plane motion is present $[17,18]$.

With an average thickness of the myocardial wall of 8 to $15 \mathrm{~mm}$ and the limited spatial resolution of MRI sequences, the contribution of blood signal resulting from partial volume effects at the interface between the cavity and the myocardium may affect MR thermometry. Blood suppression techniques could reduce this effect. The resulting increased contrast between the cavity and the myocardium may also be beneficial for the robustness of motion estimation algorithms, which are based on the analysis of local intensity changes.

In this paper, we evaluated the feasibility of $3 T$ PRFS MR thermometry of the myocardial wall using accelerated gradient-echo sequences adapted to cardiac imaging. We explored two options for PRFS MR cardiac thermometry sequences: gradient-echo with parallel imaging and EPI readout acceleration, and gradientecho with parallel imaging only. Furthermore, three different blood suppression techniques were tested: multi-slice double inversion recovery (MDIR) [19,20,21], motion-sensitized driven equilibrium (MSDE) [22-24], and inflow saturation (IS) [25]. An assessment of the applied sequences and the blood suppression techniques was performed on MR images from eight healthy volunteers using the contrast-to-noise (CNR) and signal-to-noise (SNR)-ratios. Finally, the temporal stability of PRFS MR thermometry [26] as well as the temporal average of temperature were evaluated.

\section{Materials and Methods}

\section{Choice of Imaging parameters}

The following imaging protocol has been optimized in order to meet certain requirements for cardiac thermometry in the myocardium:

- sufficient volume coverage with spatial resolution in the $\mathrm{mm}$ range to visualize 
the temperature distribution in the myocardium

- $\mathrm{TE} \sim \mathrm{T} 2^{*}$ for optimal temperature precision using PRF thermometry [27]

- a SNR of at least 10 to guarantee sufficient phase precision

- short acquisition time per slice (<300 ms) in order to avoid intra-scan motion

Parallel imaging acceleration (SENSE) was used in order to reduce the imaging matrix, but limited by the number of coil elements and signal considerations.

With acquisition times of $\sim 150 \mathrm{~ms}$ and $\sim 220 \mathrm{~ms}$ for TFE-EPI and TFE respectively, a minimum of 3 slices per cardiac cycle could be acquired without a compromise on the spatial resolution. Due to time constraints an in-plane resolution of $3.5 \mathrm{~mm}^{2}$ was chosen and signal considerations led to the selection of a $8 \mathrm{~mm}$ slice R3.1 thickness. Even though fat suppression is necessary for PRF thermometry in organs like liver or kidney, this is not the case in the myocardium, as the fat content and chemical shift effects are negligible. However, the signal from subcutaneous fat in the field of view resulted in considerable artifacts in the center of the field of view due to the SENSE reconstruction. Therefore, fat suppression R3.2 was applied for all experiments.

\section{Imaging protocol}

Imaging was performed on a 3T MR system (Achieva, Philips, Best, The Netherlands) using a 6-channel surface cardiac coil. Respiratory through-plane motion was compensated with a slice-tracking technique that used a pencil-beam navigator placed on the right diaphragm to adjust the image slice position in realtime. All acquisitions were VCG-triggered, with a trigger delay $\left(T_{D}\right)$ set to the longest available value (between $109 \mathrm{~ms}$ and $699 \mathrm{~ms}$ ) depending on the used blood suppression technique and the cardiac frequency of the volunteer, ranging from 50 to 85 beats $/ \mathrm{min}$. For effective fat suppression in the presence of $B_{1}$ inhomogeneities, spectrally-selective adiabatic inversion recovery (SPAIR, inversion time $90 \mathrm{~ms}$ ) was applied before each slice acquisition. A RF-spoiled transient field echo (TFE) sequence $\left(T_{R}=7.3 \mathrm{~ms}, T_{E}=4.6 \mathrm{~ms}\right.$, flip angle $=26-30^{\circ}$, bandwidth/pixel $=291 \mathrm{~Hz}, \mathrm{FOV}=350 \times 280 \times 8 \mathrm{~mm}^{3}$, matrix $=100 \times 80$ ) with 
parallel imaging acceleration (SENSE) of 4 was tested.

In order to further accelerate the acquisition while maintaining a reasonable echo time for MR thermometry phase accumulation, a RF-spoiled TFE-EPI sequence $\left(T_{R}=11 \mathrm{~ms}, T_{E}=6 \mathrm{~ms}\right.$, flip angle $=26-30^{\circ}$, bandwidth $/$ pixel $=217 \mathrm{~Hz}, F O V=350$ $\times 350 \times 8 \mathrm{~mm}^{3}$, matrix $=100 \times 100$ ) with an echo train length of 3 and a SENSE factor of 3 was chosen in a second experiment. Echo train lengths of 5 or more were tested, but, despite the use of a localized main magnetic field $\left(B_{0}\right)$ shim on the heart, they led to significant image quality degradation on the heart and were not further used. In total, 100 consecutive short axis (mid position) images were acquired dynamically (dynamic scan time $t_{d y n}=0.75-1.2 \mathrm{~s}$ ) covering 3 slices per cardiac cycle (slice gap $0.8 \mathrm{~mm}$ ). The timing of the described sequence within the cardiac cycle is depicted in Fig.1a.

\section{Blood suppression techniques}

\section{Multi-slice double inversion recovery (DIR)}

The double inversion recovery (DIR) technique has been proven to reliably suppress the signal of blood, independent of the blood flow rate [19]. Nevertheless, the inversion delay severely prolongs the actual acquisition time and therefore DIR is generally limited to single-slice acquisitions, even though multislice implementations have been presented recently [20, 21].

In this work, a standard double inversion recovery (DIR) preparation module was adapted to multi-slice imaging (MDIR). As a compromise between volume coverage (a minimum of 3 slices) within the given cardiac cycle and blood suppression efficiency, inversion times $\left(T_{11} T_{12}\right)$ of $4 \mathrm{~ms} / 320 \mathrm{~ms}$ were used.

Inflow saturation (IS)

For inflow saturation, saturation slabs positioned parallel to the image stack were used to suppress the signal of inflowing spins. IS is adapted to VCG-triggered 
multi-slice cardiac imaging, and was shown to provide efficient suppression of inflowing spins [25]. Here, inflow saturation (IS) was accomplished by placing one saturation slab of 60 to $80 \mathrm{~mm}$ thickness between the imaged slices and the base of the heart at a distance of 5 to $15 \mathrm{~mm}$ leading to a maximal increase in acquisition time of $6 \mathrm{~ms}$.

\section{Motion sensitized driven equilibrium (MSDE)}

The motion sensitized driven equilibrium magnetization preparation $[22,23]$ has been evaluated and successfully used for black blood carotid imaging [24]. This approach only adds a minimal delay before the acquisition and is compatible with VCG-triggered cardiac multi-slice imaging. There is only a slight increase in scan time, but the preparatory module introduces $T_{2}$ weighting, which leads to additional signal loss. Moreover, MSDE may be sensitive to inhomogeneities of the RF excitation $\left(B_{1}\right)$ field, leading to a spatially varying flip angle, and eddy current effects, especially at high magnetic fields. The blood suppression MSDE preparatory module which was implemented for this work is detailed in Fig. $1 \mathrm{~b}$ and represents an improved version of the module proposed in [28]. In order to achieve a higher robustness against $B_{1}$ inhomogeneities, two composite inversion pulses were used. Adiabatic pulses were tested. However, the resulting images showed motion artifacts as a result of the significantly increased duration of the preparation module and thus, non-adiabatic pulses were employed. Bipolar gradients were used in order to compensate for eddy current effects, with amplitudes calculated following the definition proposed by Nguyen et al [23]

$v_{\text {enc }}=\pi \gamma / m_{1}$ where $y$ is the gyromagnetic ratio, $m_{1}$ is defined as the first moment of the applied gradient, and $v_{\text {enc }}$ corresponds to the velocity of the blood spins which are dephased by $\pi$. This blood suppression technique is therefore velocity selective and an adaptation of $v_{e n c}$ is required for each volunteer in order to match the blood velocity. The value of $v_{\text {enc }}$ was adjusted on each volunteer between 20 $\mathrm{cm} \cdot \mathrm{s}^{-1}$ and $40 \mathrm{~cm} \cdot \mathrm{s}^{-1}$ (corresponding to $\mathrm{m}_{1}$ of $16 \mathrm{mT} \cdot \mathrm{m}^{-1} \cdot \mathrm{ms}$ to $8 \mathrm{mT} \cdot \mathrm{m}^{-1} \cdot \mathrm{ms}$ ). It was adapted by varying the gradient strength while the length of the preparation 
module remained fixed at $9 \mathrm{~ms}$.

No precise adjustment of $v_{e n c}$ for the three different encoding directions was performed as such an approach was considered too time-consuming.

The time penalties per image stack resulting from the application of one of the described blood suppression techniques were $320 \mathrm{~ms}$ for MDIR (corresponding to $T_{12}$ ), $9 \mathrm{~ms}$ for MSDE and $6 \mathrm{~ms}$ for IS.

\section{Image Processing}

\section{Motion compensation}

Despite the use of slice tracking and VCG triggering, which resulted in a substantial reduction of heart motion, residual in-plane displacements could be observed. In-plane motion compensation was performed by analyzing local displacements on the magnitude images. For this purpose, the first image in the time series was selected as the reference image, on which a region of interest (ROI) delimiting the left myocardium was manually drawn. A gradient driven descent algorithm maximizing the inter-correlation coefficient between this reference image and each following magnitude image of the time series was performed, assuming an affine displacement restricted to the selected ROI [17]. The 6 coefficients of the affine transformation (two translations, two scales, a rotation, a shear) leading to the highest correlation with the reference image were stored for each image of the time series. Images containing artifacts due to erroneous navigator slice tracking were identified by calculating the Pearson intercorrelation coefficient $\mathrm{C}_{0, \mathrm{n}}$ between each image and the first image of the time series. A rejection criterion was introduced such that images with $\mathrm{C}_{0, \mathrm{n}}<0.7$ were removed for further evaluation. In 7 of the 64 dynamic imaging series (100 images each), 2 to 27 images (on average 9 images) containing artifacts from erroneous slice tracking were detected and removed.

Evaluation of the image quality 
In order to evaluate the efficiency of the different blood suppression techniques SNR and CNR were evaluated from the motion compensated images. The inhomogeneous noise distribution in the images as a result of the applied parallel imaging technique required a modified definition for both expressions [24]

$$
\begin{aligned}
S N R & =\frac{S_{\mathrm{sep}}}{\sigma_{\mathrm{sep}}} \\
C N R & =\frac{S_{\mathrm{sep}}-S_{\mathrm{cav}}}{0.5\left(\sigma_{\mathrm{sep}}+\sigma_{\mathrm{cav}}\right)}
\end{aligned}
$$

where $\mathrm{S}_{\text {sep }}$ denotes the average signal in a small $\mathrm{ROI}$ in the ventricular septum, $S_{\text {cav }}$ the average signal in a small ROI in the cavity and $\sigma_{\text {sep }}$ and $\sigma_{\text {cav }}$ the respective signal standard deviations in the same ROls. As a result, the calculated SNR allowed to draw conclusions about the ratio between the measured signal and the corresponding spatial variation of the signal in the chosen ROI, but not about the image noise in general.

\section{Temperature calculation}

Susceptibility effects were corrected with a model-based multi-baseline approach [17]. The first 50 images were acquired in a learning phase and the coefficients calculated during the registration were stored and used to create parametric phase maps assuming a linear relationship between respiratory motion and susceptibility related phase changes [29]. These parametric phase maps were computed by a Singular Value Decomposition (SVD) of the list of motion registered acquired phase images with the coefficients. For the following 50 images, the coefficients retrieved during the image registration and the parametric phase maps derived during the learning phase were used to compute a synthetic phase image. This synthesized reference phase was subtracted from the current phase to calculate the temperature map using the PRFS method [11]. In order to assess the quality of the temperature measurements in the myocardium, the temporal mean $\mu_{T}$ (reflecting systematic offsets) and standard deviation of temperature $\sigma_{\mathrm{T}}$ (reflecting precision and stability) were calculated at each pixel. As rapid gradient echo- 
based sequences are prone to artifacts at the interfaces between myocardium, liver and lungs, the central region of the myocardium, corresponding to the ventricular septum, was chosen as target area for a detailed statistical analysis. The spatial distribution of $\sigma_{T}$, as well as the spatial average of $\sigma_{T}$ in a ROI in the ventricular septum $\left\langle\sigma_{T}\right\rangle$, as well as the spatial distribution of the temporal mean of temperature $\mu_{T}$ and its spatial average $\left\langle\mu_{T}>\right.$, were analyzed. The aim of this evaluation was to find a combination of imaging sequence and blood suppression technique providing a minimal temporal standard deviation of temperature $\sigma_{T}$ (corresponding to a maximal temperature stability) as well as a minimal temporal mean of temperature $\mu_{\mathrm{T}}$. For this evaluation, all dynamic images of one slice for the 8 volunteers (in total 800 images) were used for each combination of acquisition sequence and blood suppression technique.

\section{Statistical Analysis}

The significance of the results for the SNR/CNR, and-the temperature stabilities, and temporal averages of temperature for the different blood suppression techniques was evaluated using ANOVA (analysis of variances) in form of a F-test with significance threshold $p=0.05$ for each volunteer separately. For this, ANOVA (analysis of variances) in form of a F-test was performed on the results for all blood suppression techniques with significance threshold 0.05 -For the temperature stability and the temporal average of temperature, all voxels within the ventricular septum were taken into account and the average values across volunteers were

compared between the different blood suppression techniques. The significance of $R 2.1, R 2.2$, the result for CNR and SNR was evaluated by comparing the average values across patients for each blood suppression method. If the test was found significant, additional paired t-tests were applied to the data of all pairs of blood suppression techniques for the respective volunteer. A significance of $p=0.05$ was used and corrected with the Bonferroni method. This statistical analysis was performed slice by slice. 
Results

\section{$\underline{\text { TFE sequence }}$}

\section{SNR/CNR and image quality}

Figure 2a-d show typical magnitude images of the central slice obtained on the same volunteer without blood suppression and using MDIR, MSDE and IS (from left to right). All three blood suppression techniques in combination with TFE showed a comparable good performance in terms of blood suppression efficiency and image quality. As a criterion for the evaluation of the blood suppression performance, the mean value over all volunteers of the temporal average of the CNR and SNR in the ventricular septum calculated according to equation (1) together with the corresponding temporal standard deviation are displayed in Fig. 3. The application of any of the three blood suppression techniques led to an increased CNR between the septum and the cavity without significant difference between the three blood suppression techniques $(p>0.05)$. For each volunteer, the achieved average CNR for every blood suppression method was compared with $\mathrm{R} 2.3$ the other three methods. On average, MDIR showed a CNR which was approximately $30 \%$ higher than for MSDE or IS. Furthermore, iln the acquisitions without blood suppression, for 6 of 8 volunteers the myocardium appeared hypointense in comparison to the signal of the blood in the cavity leading to a negative CNR (results not shown). On average, all blood suppression techniques led to an increased SNR in the ventricular septum as compared to the acquisition without blood suppression, but For the SNR, no significant difference between the blood suppression techniques could be observed $(p>0.05)$. According to equation (1), a high SNR is an indication of reduced spatial signal variation in the septum as a result of the applied blood suppression. For the two remaining slices (results not shown), the results were comparable for the acquisitions without blood 
techniques in the last slice. Inflow saturation produced a much lower CNR in the first slice than in slices 2 and 3 . However, inflow saturation produced a much lower CNR in the first slice than in slices 2 and 3, while the other three blood suppression techniques delivered comparable CNR's for each slice. In all three slices, the application of blood suppression led to an increased CNR $(p<0.05)$.

\section{Temperature stability and temporal mean of temperature}

As a second comparison criterion, the results of the evaluation of the temperature stability and the temporal mean of temperature in the myocardium are shown in Figures $4 a-d$ and $5 a-d$ as an overlay on the magnified magnitude images for the central slice. Note that the distributions of the temperature stability and the temporal means of temperature were spatially inhomogeneous, showing a degradation at the interface with the liver and the lungs intersection of the R3.9 myocardium, liver, and lungs. However, at the interface between the lung and the myocardium and in the ventricular septum the temperature stability distribution and the distribution of the temporal mean of temperature remained stable. In the ventricular septum a temperature stability of $2.8^{\circ} \mathrm{C}$ could be achieved with the TFE $\mathrm{R} 2.3$ sequence. A detailed evaluation of the spatial distribution of the temperature stability in the ventricular septum is presented in Fig. 6 for the central slice using the combined data from all volunteers in form of Box-and-Whisker plots. In addition, the spatial average of the temperature stabilities in the ventricular septum including the corresponding spatial standard deviations are shown in Table 1 as the mean, minimal and maximal value over all volunteers for the different blood suppression techniques. Compared to the acquisition without blood suppression, the temperature stability was enhanced in 4 out of 8 cases for MDIR, in 5 out of 8 cases for IS and in 6 out of 8 cases for MSDE $(p<0.05)$, but did not show a significant improvement for MDIR ( $p>0.05$, data not shown). Among the different $\mathrm{R} 2.1, \mathrm{R} 2.2$, $\mathrm{R} 2.3$ blood suppression methods, MSDE achieved the best temperature stability $(p<0.05)$. In the cases with reduced temperature stability as compared to the results achieved without blood suppression, especially high deviations were found 
for MDIR for 2 volunteers, for MSDE for 3 volunteers, and for IS for 3 volunteers. No obvious correlation between the measured CNR in the ventricular septum as shown in Fig. 3 and the temperature stabilities were found.

Finally, Table 1 shows the spatial average and standard deviations of the temporal mean of temperature in the ventricular septum as the mean, minimal and maximal value over all volunteers for all blood suppression methods. On average, a positive temperature offset of $0.0^{\circ} \mathrm{C}$ to $0.7^{\circ} \mathrm{C}$ has been measured without a statistically $\mathrm{R} 3.12$ significant difference between the blood suppression methods (ANOVA $p=0.05 p>0.05$ ).

For the two remaining slices the results for the temporal average of temperature and temperature stability were comparable. All blood suppression techniques showed a decreased temperature stability in the first and last slice, only MDIR R2.1,R2.2, showed a decreased temperature stability in the last slice.

In general, the average blood suppression performance of the three blood suppression techniques was comparable.

\section{$\underline{\text { TFE-EPI sequence }}$}

\section{SNR/CNR and image quality}

The images acquired using MSDE and IS showed a comparable quality and blood suppression efficiency, whereas the image acquired with MDIR suffered from motion-induced blurring (Fig. 2e-h). MDIR and MSDE suffered from a reduced or comparable SNR in the ventricular septum as compared to the acquisition without blood suppression, while IS showed a higher SNR $(p<0.05)$ indicating that IS achieved a more favorable ratio between myocardial signal and spatial signal homogeneity than MDIR and MSDE (Fig. 3 right). As expected, the CNR between R2.1,R2.2, the myocardium and the cavity was increased for all blood suppression techniques, with the IS technique performing best in comparison with MDIR and MSDE $(p<0.05)$. Furthermore, in the acquisitions without blood suppression for 6 
of 8 volunteers and for MSDE for 2 volunteers, the myocardium appeared hypointense in comparison to the signal of the blood in the cavity leading to a negative CNR, as illustrated in Fig. 3 displaying the average CNR(data not shown). For the remaining slices (results not shown) a similar behavior was found the differences between the different methods were reduced and Only IS showed a decreased CNR in the first slice.

\section{Temperature stability and temporal mean of temperature}

Similar to the acquisitions using TFE without EPI readout, the temperature stability and temporal mean of temperature were deteriorated at the interface with the lungs and the liver intersection of the myocardium, liver, and lungs, but improved in the other sections of the myocardium (Fig. 4e-h and 5e-h). Despite the apparent blurring in the magnitude images for MDIR, the measured temperature stability R3.6 and temporal mean of temperature in the myocardium were not affected by these artifacts. A detailed statistical analysis of the spatial distribution of the temperature stability in the ventricular septum for the different blood suppression techniques is shown in Fig. 6 and Table 1 using the combined data from all volunteers and the central slice. Statistically significant improvements of the temperature stability in comparison to the acquisition without blood suppression could be observed for all three blood suppression techniques in 3 of 8 cases for MSDE, in 5 of 8 cases for R2.1,R2.2,

MDIR, and in 7 of 8 cases for is $(p<0.05)$ (data not shown). Unlike the $\mathrm{R} 2.3$ acquisitions using TFE without EPI readout, IS blood suppression performed significantly better than MDIR in 5 of 8 cases and than MSDE in 7 of 8 cases $(p<$ 0.05), as confirmed in the Box-and-Whisker plots in Fig. 6, which represents the combined evaluation over the ROl's of all volunteers. Among the cases with reduced temperature stability as compared to the acquisition without blood suppression, remarkable poor temperature stabilities were calculated for MDIR for 1 volunteer, and for MSDE for 3 volunteers. This manifested itself also in substantially decreased average temperature stabilities, as presented in Table 1, where for the mentioned cases values of up to $16.9^{\circ} \mathrm{C} \pm 22.9^{\circ} \mathrm{C}$ could be observed. 
For this sequence, there was a good correspondence between the observed CNR in the ventricular septum as displayed in Fig. 3 and the corresponding temperature stability for the majority of cases. This was confirmed in the results of MSDE for 2 volunteers, where a hypo-intense signal in the septum was coupled to a significantly reduced temperature stability. A similar observation could be made for 7 of 8 volunteers in the cases where no blood suppression was applied. On the other hand a significantly increased CNR for 4 volunteers was coupled to a very high temperature stability in the ventricular septum (data not shown).

Figure 7 shows an example graph of the temperature measured in three different characteristic points in the ventricular septum for the IS technique and volunteer 5, central slice. The temperature remained stable and was shifted by approximately $0.7^{\circ} \mathrm{C}, 1.5^{\circ} \mathrm{C}$ and $1.9^{\circ} \mathrm{C}$ for the three different points. The temperature graphs confirm that the temporal standard deviation of temperature increased for the points closer to the interface with the lungs and the liver as already stated above. In addition, Table 1 shows the spatial average and standard deviation of the R3.7 temporal mean of temperature in the ventricular septum as the mean, minimal and maximal value over all volunteers for all blood suppression techniques. On average, mean temperatures of $0.2^{\circ} \mathrm{C}$ to $0.4^{\circ} \mathrm{C}$ have been measured for the different blood suppression techniques. The last acquired slice showed similar temperature stabilities and temporal averages of temperature as the central slice. For the first slice, the acquisitions with IS and MSDE produced lower temperature stabilities than for the second and third slice.

\section{Discussion}

\section{Imaging protocol}

A direct comparison of the two applied sequences (TFE and TFE-EPI) is not straightforward as several factors have to be taken into account. The TFE sequence was robust against motion artifacts and $B_{0}$ inhomogeneities while suffering from longer acquisition times. As a result, the achievable echo times 
were limited, leading to a reduced temperature precision. Combining this sequence with an EPI readout led to a significant acceleration and reduced intrascan motion, but the EPI readout was intrinsically more sensitive to susceptibility artifacts. The TFE-EPI sequence achieved on average better temperature stabilities in the ventricular septum, and enabled higher volume coverage and longer echo times.

The applied imaging protocol for MR thermometry required a compromise between spatial resolution, volume coverage, overall scan time and signal. In this study, a R3.7 spatial resolution of $3.5 \times 3.5 \times 8 \mathrm{~mm}^{3}$ covering 3 slices was chosen in order to allow a temporal resolution of one heart beat, yielding an average SNR of 15 . Considering the size of a catheter tip and the expected hot spot during a RF ablation procedure, a higher spatial resolution would be desirable. As the SNR directly influences the theoretically achievable temperature precision [26], a further reduction of the voxel size would result in a decreased temperature precision, if the associated signal loss is not compensated by other means. A potentially higher signal could, for example, be achieved by using available 32-channel cardiac coils. Increasing the SENSE acceleration factor could be beneficial, but again, the resulting signal loss would have to be compensated in order to maintain a sufficient temperature precision. Furthermore, longer echo times, corresponding to potentially higher temperature precisions and increased volume coverage, could be achieved by selecting smaller fields of view (FOV). However, established inner volume excitation techniques [30,31] are usually based on inversion techniques which are not compatible with gradient echo imaging for MR thermometry. Other approaches using tailored RF pulses have been successfully tested, for example in $3 \mathrm{D}$ carotid imaging [32], but have not yet been applied to MR temperature imaging. The use of saturation slabs for the FOV reduction is possible with gradient echo sequences, but leads to an increased acquisition time.

\section{Blood suppression techniques}

IS: In terms of blood suppression, inflow saturation appeared to be the most robust 
method yielding a spatial average temperature stability in the ventricular septum of $2^{\circ} \mathrm{C}$ or better when combined with the TFE-EPI acquisition. Nevertheless, the application of inflow saturation slabs remained time-consuming and care had to be taken to avoid saturation of the spins included in the volume excited by the pencil beam. Furthermore, as IS is based on the inflow effect, the blood suppression efficacy in every slice depended on the distance of the saturation slab from the image stack, the exact blood velocity, and the slice order. In our setup, blood suppression was always more effective in the last acquired slice.

MDIR: Multi-slice DIR achieved good blood suppression over all slices leading to an average temperature stability of $3^{\circ} \mathrm{C}$ when used with a TFE sequence. However, it suffered from long scan times as a result of the necessary inversion delay limiting the slice number to 2 to 3 for typical cardiac frequencies (ranging from $0.8 \mathrm{~Hz}$ to $1.2 \mathrm{~Hz}$ for the presented volunteers). Furthermore, in our implementation, the combination with EPI readout led to severe image artifacts. This may be due to the fact that the EPI phase correction data was acquired prior to the inversion delay. It is acquired once at the beginning of a dynamic series of images by switching off the phase encoding gradients. However, if motion occurs between the acquisition of the phase correction data and the acquisition of the actual image, the resulting images will contain artifacts due to uncompensated residual phase offsets between the acquired k-space lines. A catheter tip in contact with the myocardium is likely to cause additional susceptibility artifacts, and hence lead to a further deterioration of the image quality [33]. If these problems are resolved, multi-slice DIR could become a candidate for blood suppression during MR thermometry using TFE sequences with or without EPI acceleration.

MSDE: The application of MSDE for blood suppression resulted in an average temperature stability of $3^{\circ} \mathrm{C}$ or better. However, due to its sensitivity to $B_{0}$ and $B_{1}$ inhomogeneities, large variations in the blood suppression performance were observed. This is also because for a chosen gradient strength, only one particular blood velocity is completely suppressed. As this technique also reduces the signal 
from all bulk motion, in some cases, the signal of the myocardium was reduced as well, leading to a degraded CNR between myocardium and cavity. General signal loss in the myocardium may be overcome by applying $B_{1}$ shimming [34] prior to the measurement, and by choosing different gradient amplitudes in the three encoding directions.

The results confirmed that blood suppression is beneficial for MR thermometry of the heart as indicated by the improved temperature stability in the myocardium. This is most likely due to a combination of reduced partial volume effects at the myocardium-blood interface and to a better performance of the motion correction algorithms when presented with images with improved myocardium-cavity delineation. The observed average temperatures showed a large spatial variation and represent thus no systematic but random temperature offset.

Based on the average temperature stabilities and SNR/CNR values in the ventricular septum, IS in combination with TFE-EPI was the preferred combination for the presented implementation.

\section{Image Quality}

The measured SNR/CNR values in the ventricular septum indicated that the applied blood suppression was able to significantly increase the CNR between the myocardium and the cavity. It was also found that the application of blood suppression typically leads to an increased SNR in the ventricular septum. As all blood suppression methods are designed to cancel the signal of moving spins whilst also tissue spins may not remain completely unaffected, this result seems counterintuitive. This result may be attributed to a decreased spatial standard deviation of the signal, potentially as a result of reduced partial volume effects with blood and reduced flow artifacts.

\section{Motion correction}

The applied affine algorithm for the compensation of in-plane motion was still 
limited when applied in the myocardium, as already small deformations or residual flow effects could cause false pixel displacements in the myocardium. The temperature calculation during RF heating may be severely biased by the resulting false displacements. A local motion estimation algorithm [35] may be helpful, but may produce false corrections when presented with insufficient flow suppression. Hence, the development of more elaborated motion correction techniques will be the subject of future work. In 7 of the 64 dynamic imaging series (100 images each), 2 to 27 images (on average 9 images) containing artifacts from erroneous slice tracking were detected and removed. The removal of images containing artifacts represented a necessary processing step since Wwithout image rejection, temporal standard deviations of temperature above $20^{\circ} \mathrm{C}$ were measured proving the necessity of this processing step for accurate thermometry in the heart.

The presented results for the temperature stability in the myocardium resulted from a combination of several effects including the quality of the motion and phase correction algorithms, the accuracy of the applied navigator slice tracking technique, and the efficiency of the applied blood suppression methods. As a result, they allowed only limited statements about the respective contributions of each parameter onto the temperature stability. Finally, the conclusions of this paper are based on the precision of MR thermometry in the absence of heating. Hence, future work should further explore the presented method during RF heating.

\section{Conclusions}

PRFS-based cardiac MR-thermometry at 3T using a TFE-EPI sequence with inflow saturation blood suppression could achieve average temperature stabilities of $2^{\circ} \mathrm{C}$ or better in the ventricular septum, in combination with cardiac triggering, navigator respiratory compensation, parallel imaging, and a motion compensation and multi-baseline susceptibility correction algorithm. For this setup, inflow 
saturation by saturation slabs appeared to be the blood suppression method of choice.

\section{Acknowledgments}

This work has been supported by the DiMI (Diagnostic Molecular Imaging) network and the author received a CIFRE stipend sponsored by ANRT (association nationale recherche technologie) and Philips Healthcare France. Finally, we thank Micheal McConnell (Stanford school of medicine) for helpful discussions.

\section{References}

[1] Chugh A, Morady F. Atrial fibrillation: Catheter ablation. J. Interv. Card. Electrophysiol. 2006; 16:15-26.

[2] Jaïs P, Cauchemez B, Macle L, Daoud E, Khairy P, Subbiah R, Hocini M, Extramiana F, Sacher F, Bordacher P, et al. Catheter ablation versus antiarrhythmic drugs for atrial fibrillation: The A4 study.

Circulation 2008; 118(24):2498-2505.

[3] Ector J, de Buck S, Huybrechts W, Nuygens D, Dymarkowski S, Bogaert J, Maes F, Heidbüchel $\mathrm{H}$. Biplane three-dimensional augmented fluoroscopy as single navigation tool for ablation of atrial fibrillation: Accuracy and clinical. Heart Rythm 2008; 5:957-964.

[4] Hijazi Z, Shivkumar K, Sahn D. Intracardial echocardiography during interventional and electrophysiological cardiac catheterization.

Circulation 2009; 119:587-596. 
[5] Lacomis M, Wigginton W, Fuhrman C, Schwarzman D, Armfeld R, Pealer K. Multi-detector row $\mathrm{CT}$ of the left atrium and pulmonary veins before radiofrequency catheter ablation for atrial fibrillation.

Radiographics 2003; 23:S35-S48.

[6] Dickfeld T. Magnetic resonance imaging and radiofrequency ablations. Herzschr. Elektrophys. 2007; 18:147-156.

[7] Malchano Z, Neuzil P, Cury R, Holmvang G, Weichet J, Schmidt E, Ruskin J, Reddy V. Integration of cardiac CT/MR imaging with three-dimensional electroanatomical mapping to guide catheter manipulation in the left atrium: Implications for catheter ablation of atrial fibrillation.

J. Cardiovasc. Electrophysiol. 2006; 17:1221-1229.

[8] Saremi F, Tafti M. The role of computed tomography and magnetic resonance imaging in ablation procedures for treatment of atrial fibrillation.

Semin. Ultrasound CT MR 2009; 30(2):125-156.

[9] Schmidt E, Yoneyama R, Dumoulin C, Darrow R, Klein E, Kiruluta A, Hayase M. 3D coronary motion tracking in swine models with MR tracking catheters. J. Magn. Res. Imag. 2009; 29:86-98.

[10] Berruezo A, Ortiz-Peréz J, Guasch E, Tamborero D, Silva E, de Caralt T, Perea R, Boussy T, Mont L, Brugada J. Noninvasive evaluation of radiofrequency lesions in the human ventricular myocardium by contrastenhanced cardiac magnetic resonance. Circulation 2009; 2:208-11.

[11] Isihara Y, Calderon A, Watanabe H, Okamoto K, Suzuki Y, Kuroda K, Suzuki Y. A precise and fast temperature mapping using water proton chemical shift. Magn. Res. Med. 1992; 34(6):814-23.

[12] Seror, Lepetit-Coi $\square$ é M, Bail BL, de Senneville BD, Trillaud H, Moonen C, Quesson B. Real time monitoring of radiofrequency ablation based on MR thermometry and thermal dose in the pig liver in vivo.

Europ. Radiol. 2008; 18(2):408-16.

[13] Bundy J, Simonetti O, Laub G, Finn P. TrueFISP imaging of the heart. Proc. $7^{\text {th }}$ ISMRM, Berkeley (Calif), 1999. P 1282. 
[14] Carr J, Simonetti O, Bundy J, Li D, Pereles S, Finn J. Cine MR angiography of the heart with segmented true fast imaging with steady-state precession. Radiology 2001; 219:828-834.

[15] Scheffler K. Fast frequency mapping with balanced SSFP: Theory and Application to Proton Resonance Frequency Shift Thermometry. Magn. Res. Med. 2004; 51:1205-1211.

[16] Nitz W, Oppelt A, Renz W, Manke C, Lenhart M, Link J. On the heating of linear conductive structures as guide wires and catheters in interventional MRI. J. Magn. Res. Imag. 2001; 13:105-114.

[17] Roujol S, Ries M, Quesson B, Moonen C, Denis de Senneville B. Real-time MR thermometry and dosimetry for interventional guidance on abdominal organs. Magn. Res. Med. 2010, 63(4):1080-1087

[18] Rieke V, Vigen K, Sommer G, Daniel B, Pauly J, Butts K. Referenceless PRF shift Thermometry. Magn. Res. Med. 2004; 51:1223-1231.

[19] Edelman R, Chien D, Kim D. Fast selective black blood MR imaging. Radiology 1991; 181:655-660.

[20] Song $\mathrm{H}$, Wright A, Wolf R, Wehrli F. Multislice double inversion pulse sequence for efficient black blood MRI. Magn. Res. Med. 2002; 47:616-620.

[21] Yarnykh V, Yuan C. Multislice double inversion-recovery black-blood imaging with simultaneous slice reinversion. J. Magn. Res. Imag. 2003;17:478-483.

[22] Wang J, Yarnykh V, Hatsukami T, Chu B, Balu N, Yuan C. Improved suppression of plaque-mimicking artifacts in black-blood carotid atherosclerosis imaging using a multislice motion-sensitized drivenequilibrium (MSDE) turbo spin-echo (TSE) sequence.

Magn. Res. Med. 2007; 58:973-981.

[23] Nguyen T, de Rochefort L, Spincemaille P, Cham M, Weinsaft J, Prince M, Wang Y. Effective motion-sensitizing magnetization preparation for black blood magnetic resonance imaging of the heart. J. Magn. Res. Imag. 2008; 28:1092-1100.

[24] Koktzoglou I, Li D. Diffusion-prepared segmented steady-state free 
precession: Application to 3D black-blood cardiovascular magnetic resonance of the thoracic aorta and carotid artery walls.

J. Cardiovasc. Magn. Res. 2007; 9:33-42.

[25] Felmlee J, Ehman R. Spatial presaturation: A method for suppressing flow artifacts and improving depiction of vascular anatomy in MR imaging. Radiology 1987; 164:559-564.

[26] Weidensteiner C, Quesson B, Caire-Gana B, Kerioui N, Rullier A, Trillaud H, Moonen C. Real-time MR temperature mapping of rabbit liver in vivo during thermal ablation. Magn. Res. Med. 2003; 50(2):322-30.

[27] de Zwart JA, van Gelderen P, Kelly DJ, Moonen CTW. Fast magneticresonance temperature imaging. J. Magn. Res. B 1996;112(1):86-90

[28] Salerno M, Epstein F, Kramer C. Diffusion-prepared dark blood delayed enhancement imaging for improved detection of subendocardial infarcts. In Proc. 12th SCMR, 2009. Vol. II(Suppl I):O10.

[29] Roujol S, Denis de Senneville B, Maclair G, Hey S, Jaïs P, Moonen C, Quesson B. Advances in real-time MR temperature mapping of the human heart. Proc. $17^{\text {th }}$ ISMRM, Hawaii, 2009. P.443.

[30] Feinberg D, Hoenninger J, Crooks L, Kaufman L, Watts J, Arakawa M. Inner volume MR imaging: Technical concepts and their application. Radiology 1985; 156:743-747.

[31] Wilm B, Gramper U, Henning A, Pruessmann K, Kollias S, Boesiger P. Diffusion-weighted imaging of the entire spinal cord.

NMR Biomed. 2009; 22(2):174-181.

[32] Bornstedt A, Bernhardt P, Hombach V, Kamenz J, Spiess J, Subgang A, Rasche V. Local excitation black blood imaging at 3T: Application to the carotid artery wall. Magn. Res. Med. 2008; 59:1207-1211.

[33] Kolandeivelu A, Zviman MM, Castro V, Lardo AC, Berger RD, Halperin HR. Non-invasive assessment of tissue heating during cardiac radiofrequency ablation using MRI thermography Circ. Arrhythm. Electrophysiol. 2010. in press. 
[34] Katscher U, Börnert P, Leussler C, van den Brink JS. Transmit sense. Magn. Res. Med. 2003; 49:144-150.

[35] Denis de Senneville B, Mougenot C, Moonen C. Real time adaptive methods for treatment of mobile organs by MRI controlled high intensity focused ultrasound. Magn. Res. Med. 2007; 57:319-330.

Figure 1: a) Illustration of the TFE sequence using the MSDE blood suppression preparation including fat suppression and pencil-beam navigator. b) Positioning of the image acquisition within the cardiac cycle (RR-interval) for a pre-defined trigger delay (TD) and 3 slices leading to a dynamic scan time $t_{d y n}$.

Figure 2: Magnified magnitude images for TFE (a-d) and for TFE-EPI (e-h) for one volunteer, central slice. From left to right: without blood suppression (noBS), MDIR, MSDE, and IS.

Figure 3: Mean value over all volunteers of the temporal average of the SNR and CNR according to equation (1) together with the corresponding standard deviations for the different blood suppression techniques and the two different sequences for the central slice. Negative CNR values indicate a hypo-intense myocardial signal as compared to the signal of the blood in the cavity. The data was retrieved from ROl's in the ventricular septum using all 800 dynamic images

R3.3, R3.5 
for each combination of acquisition sequence and blood suppression technique. The size of the ROl's varied between 95 and 294 voxels with an average value of 175 voxels.

Figure 4: Magnified magnitude images with an overlay of the temperature stability $\sigma_{T}$ for TFE (a-d) and for TFE-EPI (e-h) for one volunteer, central slice. From left to right: without blood suppression (noBS), MDIR, MSDE and IS. The data has been R3.3 retrieved from the 100 dynamic images acquired for this specific volunteer.

Figure 5: Magnified magnitude images with an overlay of the temporal average of temperature $\mu_{\mathrm{T}}$ for TFE (a-d) and for TFE-EPI (e-h) for one volunteer, central slice. From left to right: without blood suppression (noBS), MDIR, MSDE and IS. The R3.3 data has been retrieved from the 100 dynamic images acquired for this specific volunteer.

Figure 6: Box-and-Whisker plots of the spatial distribution of the temperature stability $\sigma_{T}$ in the ventricular septum for the acquisitions without blood suppression (no BS), DIR, MSDE and IS for the central slice. Shown are the values of the temperature stability which are exceeded by $10 \%, 25 \%, 50 \%, 75 \%$ and $90 \%$ of the voxels in the chosen ROI. The plots have been created from the combined data of all volunteers (in total 800 dynamic images). The size of the ROl's varied between 95 and 294 voxels with an average value of 175 voxels.

Figure 7: Example of the temperature evolution for TFE-EPI with IS for one volunteer, central slice. Shown are the graphs for three different voxels at characteristic locations in the ventricular septum as shown as white points on the magnitude image on the left.

a)

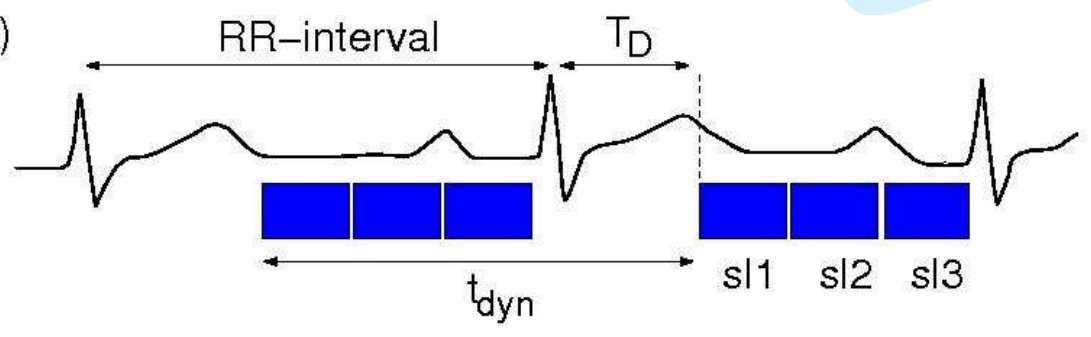

b)

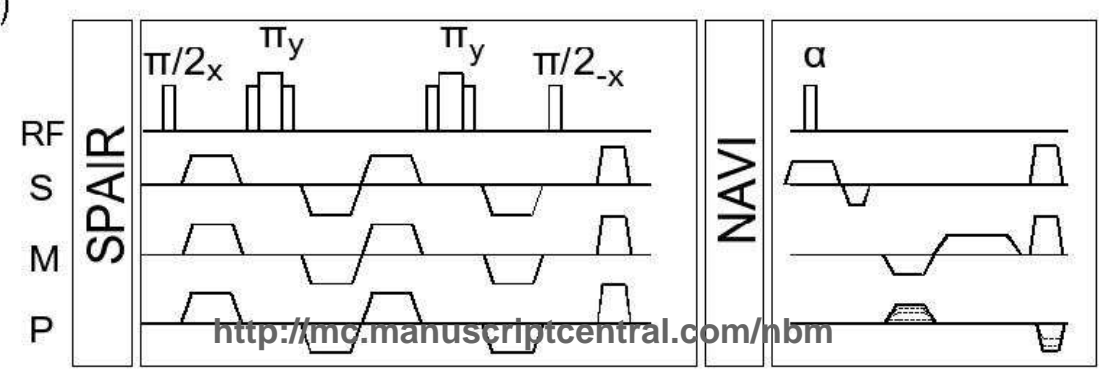


Figure 1
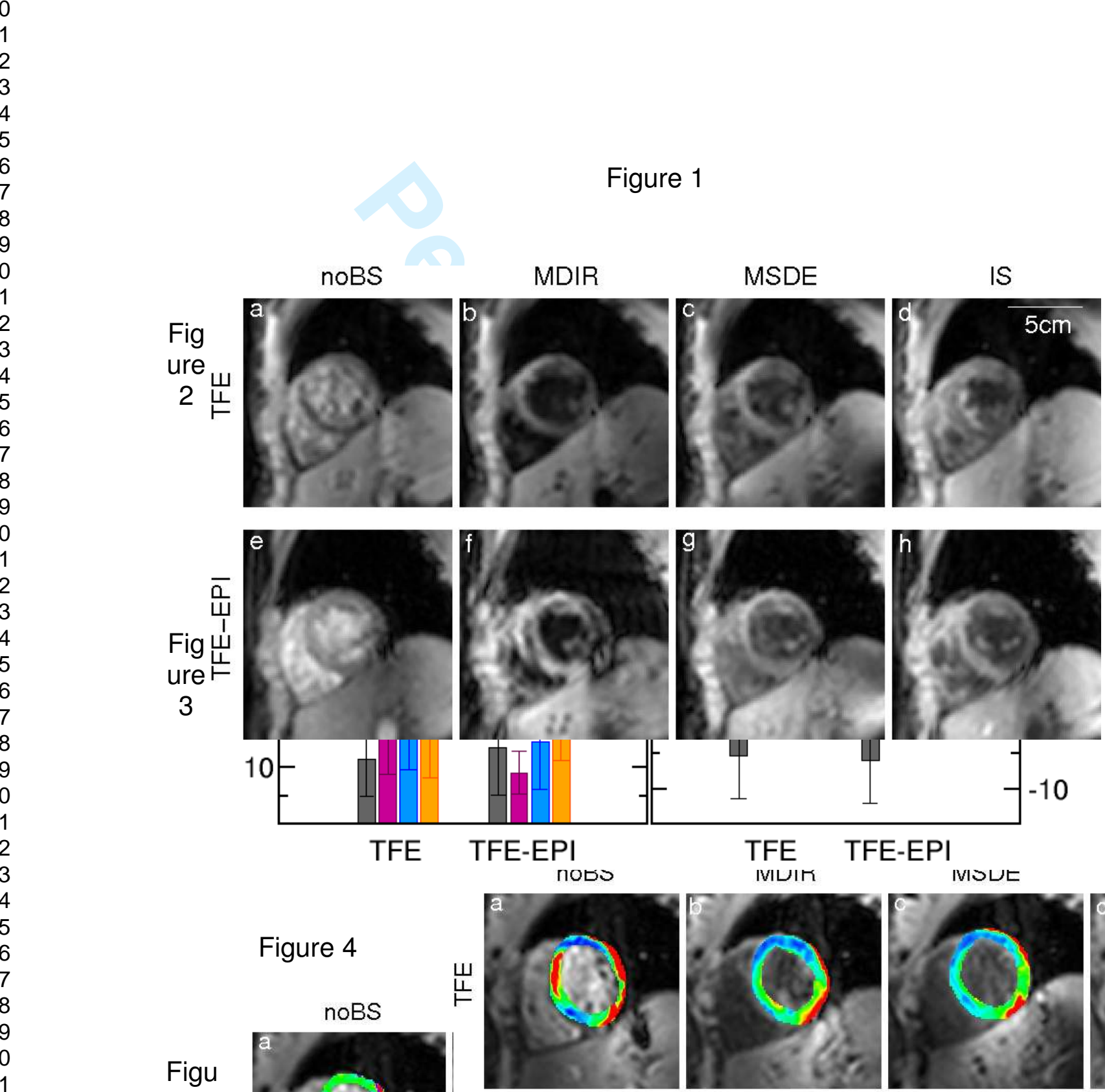

nODO IVIUIK IVISUE IS

Figure 4
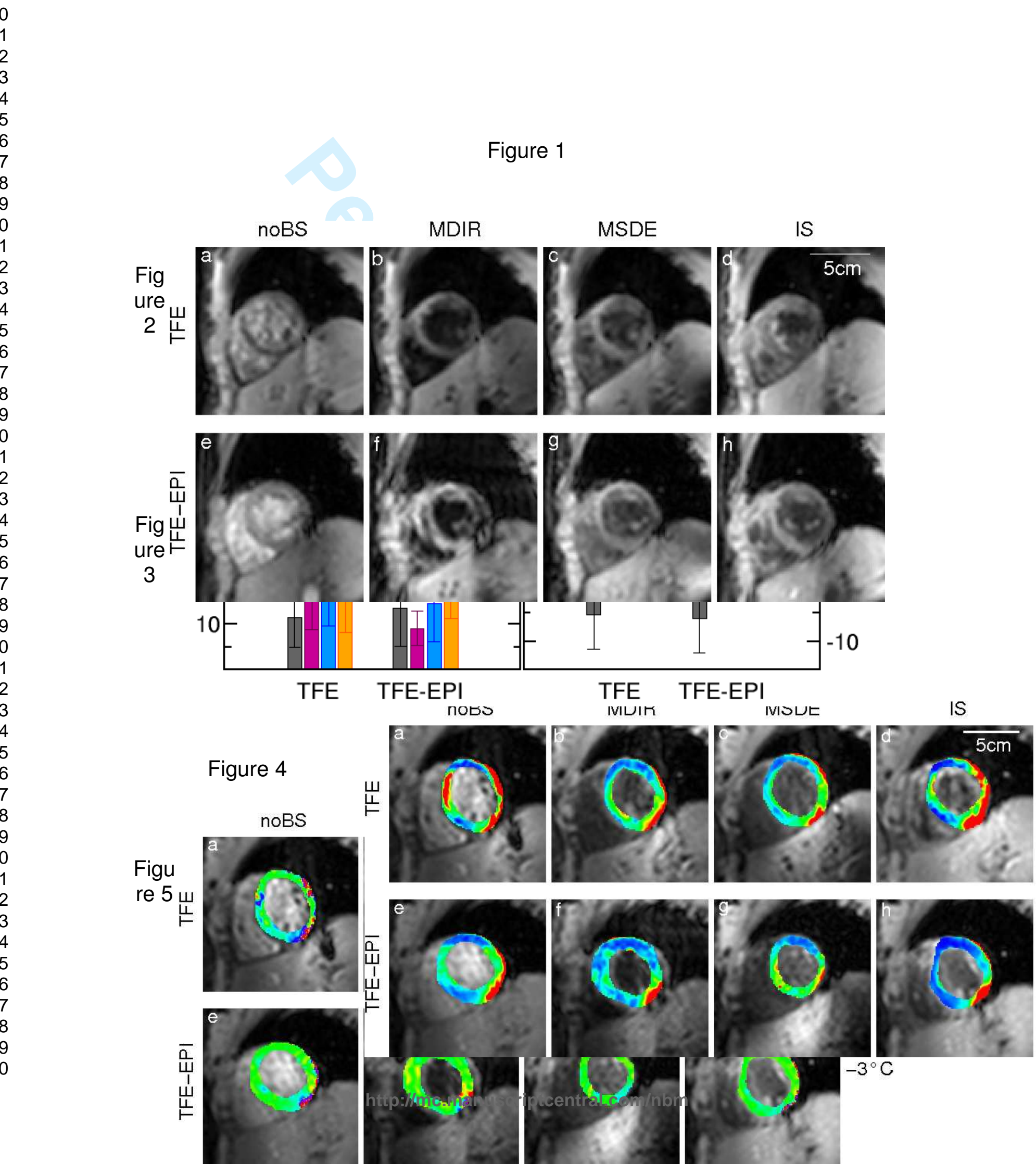

$\sigma_{\mathrm{T}}$
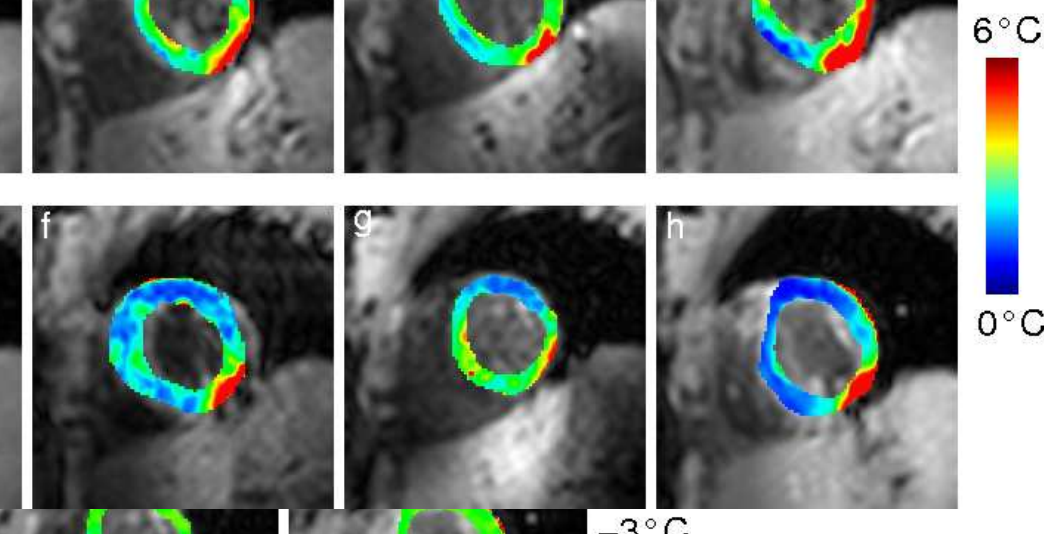


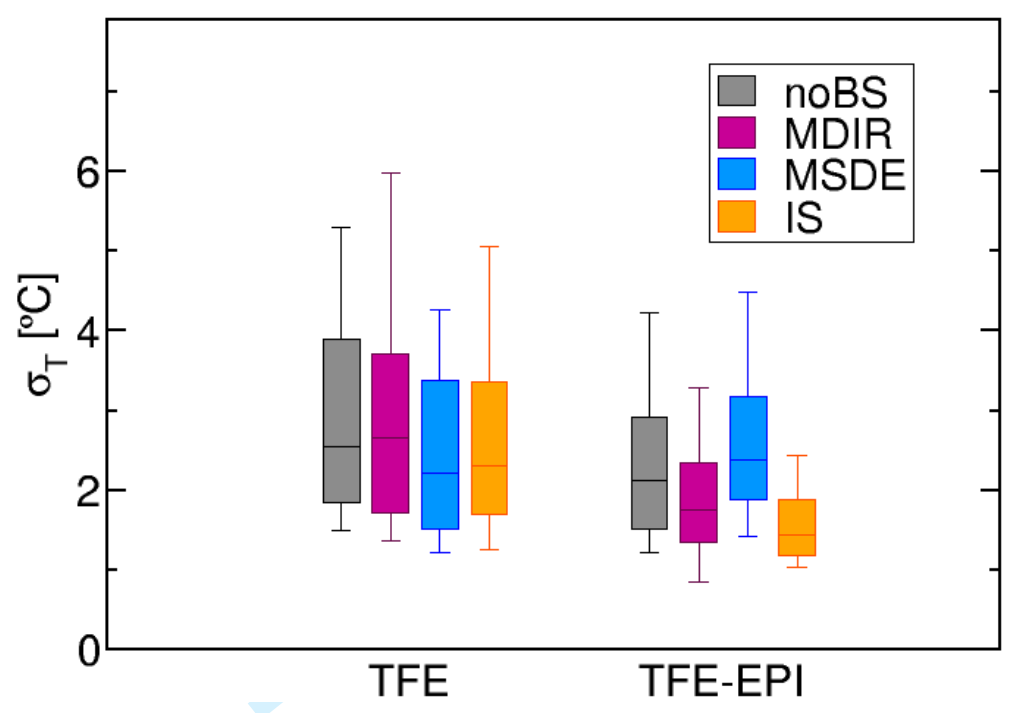

R3.11

Figure 6
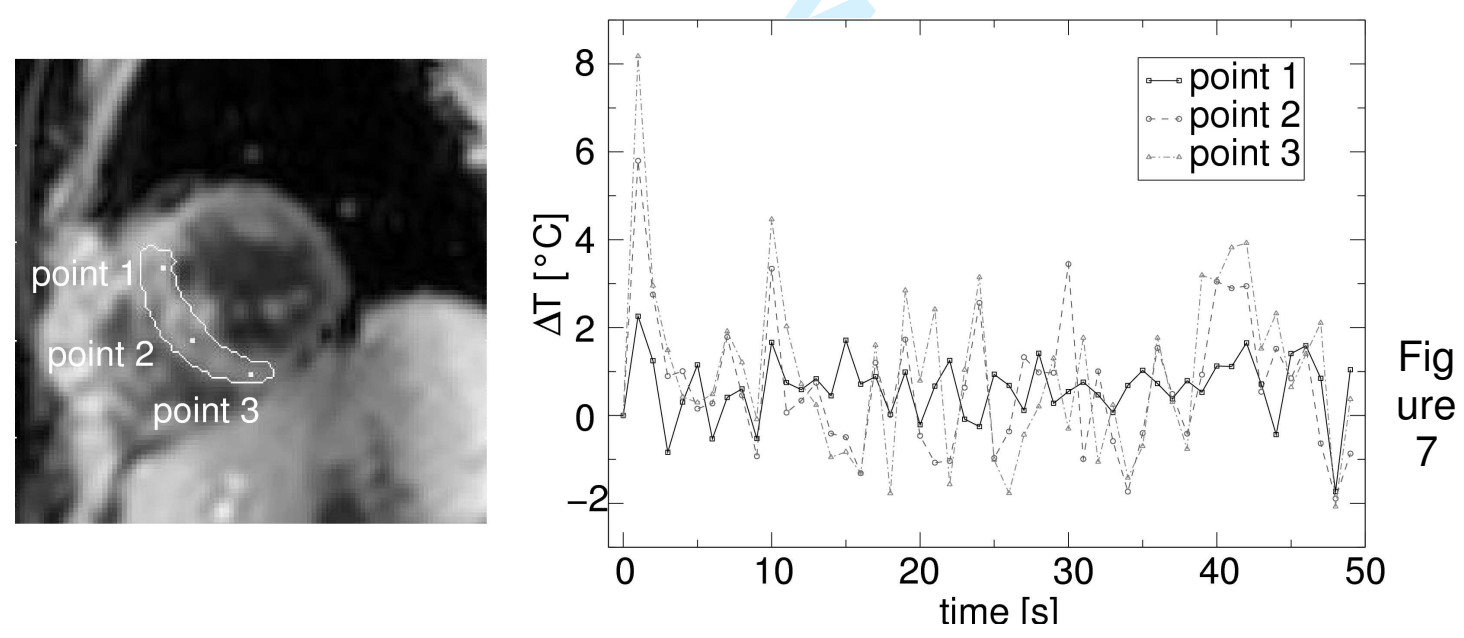

http://mc.manuscriptcentral.com/nbm 
Table 1: Spatial average of the temperature stability $\left\langle\sigma_{T}\right\rangle$ and the temporal average of temperature $\left\langle\mu_{\top}\right\rangle$ in the ventricular septum including the spatial standard deviations for all blood suppression techniques and the two tested sequences. Shown are the average values for all volunteers, as well as the minimal and maximal values found in the group of volunteers. This data is taken from the second slice. The size of the ROl's varied between 95 and 294 voxels with an average value of 175 voxels. 